\title{
Socioeconomic status and stroke incidence, prevalence, mortality, and worldwide burden: an ecological analysis from the Global Burden of Disease Study 2017
}

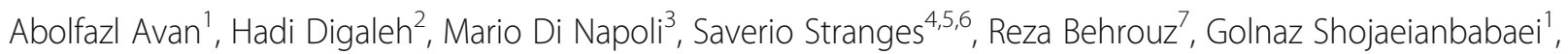
Amin Amiri ${ }^{1}$, Reza Tabrizi ${ }^{8,9}$, Naghmeh Mokhber ${ }^{10,11}$, J. David Spence ${ }^{12,13,14}$ and Mahmoud Reza Azarpazhooh 1,4,12,13*

\begin{abstract}
Background: Socioeconomic status (SES) is associated with stroke incidence and mortality. Distribution of stroke risk factors is changing worldwide; evidence on these trends is crucial to the allocation of resources for prevention strategies to tackle major modifiable risk factors with the highest impact on stroke burden.

Methods: We extracted data from the Global Burden of Diseases, Injuries, and Risk Factors Study (GBD) 2017. We analysed trends in global and SES-specific age-standardised stroke incidence, prevalence, mortality, and disabilityadjusted life years (DALYs) lost from 1990 to 2017. We also estimated the age-standardised attributable risk of stroke mortality associated with common risk factors in low-, low-middle-, upper-middle-, and high-income countries. Further, we explored the effect of age and sex on associations of risk factors with stroke mortality from 1990 to 2017.
\end{abstract}

Results: Despite a growth in crude number of stroke events from 1990 to 2017, there has been an 11.3\% decrease in age-standardised stroke incidence rate worldwide (150.5, 95\% uncertainty interval [UI] 140.3-161.8 per 100,000 in 2017). This has been accompanied by an overall $3.1 \%$ increase in age-standardised stroke prevalence rate (1300.6, UI 1229.0-1374.7 per 100,000 in 2017) and a 33.4\% decrease in age-standardised stroke mortality rate (80.5, UI 78.982.6 per 100,000 in 2017) over the same time period. The rising trends in age-standardised stroke prevalence have been observed only in middle-income countries, despite declining trends in age-standardised stroke incidence and mortality in all income categories since 2005. Further, there has been almost a 34\% reduction in stroke death rate (67.8, UI 64.1-71.1 per 100,000 in 2017) attributable to modifiable risk factors, more prominently in wealthier countries.

* Correspondence: reza.azarpazhooh@|hsc.on.ca

'Department of Neurology, Ghaem Hospital, School of Medicine, Mashhad University of Medical Sciences, Mashhad, Iran

${ }^{4}$ Department of Epidemiology and Biostatistics, Schulich School of Medicine

\& Dentistry, Western University, London, Ontario, Canada

Full list of author information is available at the end of the article

(c) The Author(s). 2019 Open Access This article is distributed under the terms of the Creative Commons Attribution 4.0 International License (http://creativecommons.org/licenses/by/4.0/), which permits unrestricted use, distribution, and reproduction in any medium, provided you give appropriate credit to the original author(s) and the source, provide a link to the Creative Commons license, and indicate if changes were made. The Creative Commons Public Domain Dedication waiver (http://creativecommons.org/publicdomain/zero/1.0/) applies to the data made available in this article, unless otherwise stated. 
(Continued from previous page)

Conclusions: Almost half of stroke-related deaths are attributable to poor management of modifiable risk factors, and thus potentially preventable. We should appreciate societal barriers in lower-SES groups to design tailored preventive strategies. Despite improvements in general health knowledge, access to healthcare, and preventative strategies, SES is still strongly associated with modifiable risk factors and stroke burden; thus, screening of people from low SES at higher stroke risk is crucial.

Keywords: Stroke, Cause of death, Global burden of disease, Global health, Non-communicable diseases, Public health practice, Risk factors, Socioeconomic factors, Life style, Morbidity

\section{Background}

Among 240 causes of death, stroke is globally the second cause of death after ischaemic heart disease [1], and it is projected to remain so by 2030 [2]. This rank varies slightly across low-income countries (LICs), lowermiddle-income countries (LMICs), upper-middleincome countries (UMICs), and high-income countries (HICs) as classified by the World Bank (Table 1) [3]. Further, stroke survivors may suffer from disabilities, requiring temporary or lifelong assistance, resulting in an enormous burden, both in human and economic costs. Evidence suggests that socioeconomic deprivation is not only associated with stroke and its risk factors, but also increases stroke severity [4] and mortality [5], and stroke incidence at younger ages [4].

Distribution of stroke risk factors in the context of socioeconomic status is changing worldwide (SES; Additional file 1: Text S1); understanding these trends is helpful in reducing the risk, through allocation of resources to those modifiable risk factors with the highest impact on stroke (Fig. 1). From 1990 to 2010, the age-standardised incidence of stroke decreased significantly by $12 \%$ in HICs, while in LMICs, it increased, although non-significantly, by about $12 \%$ [6]. However, mortality rates decreased significantly in both groups of countries (mean $37 \%$ in HICs vs. $20 \%$ in LMICs), of which $31 \%$ (with about $80 \%$ of it in LMICs) were in children and young adults (below 65 years) [6]. Further, the available evidence indicates that almost $90 \%$ of cardiovascular disease, including stroke and myocardial infarction, is caused by potentially modifiable risk factors [7]. We aimed to study the global and SES-specific stroke incidence, prevalence, burden, and mortality and to estimate the role of age, sex, and modifiable risk factors in stroke mortality.

Table 1 Age-standardised all-cause global mortality rank in 1990 and 2017 being classified by World Bank income levels (extracted from [8])

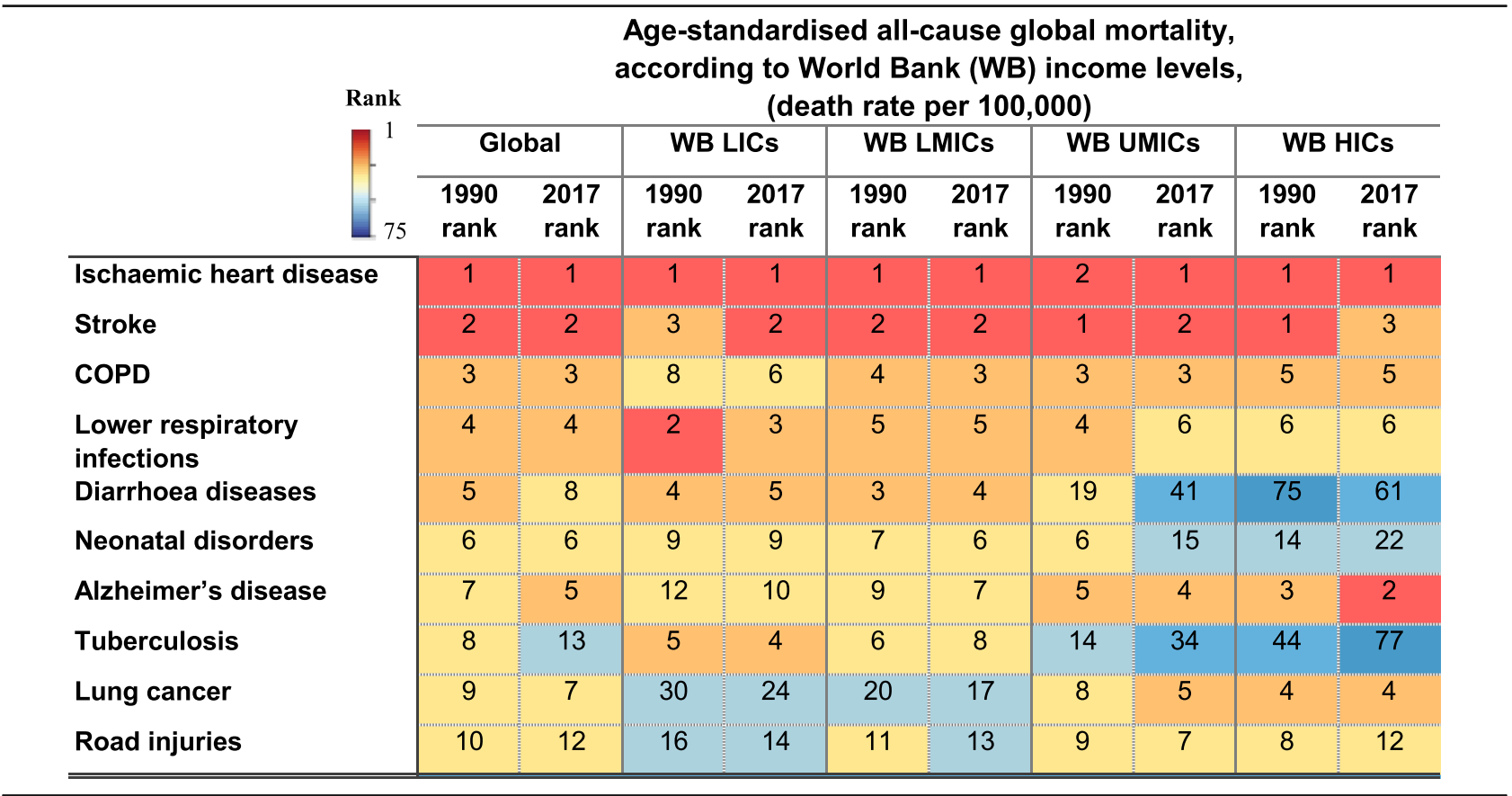

HICs high-income countries, LICs low-income countries, LMICs lower-middle-income countries, UMICs upper-middle-income countries, WB The World Bank 

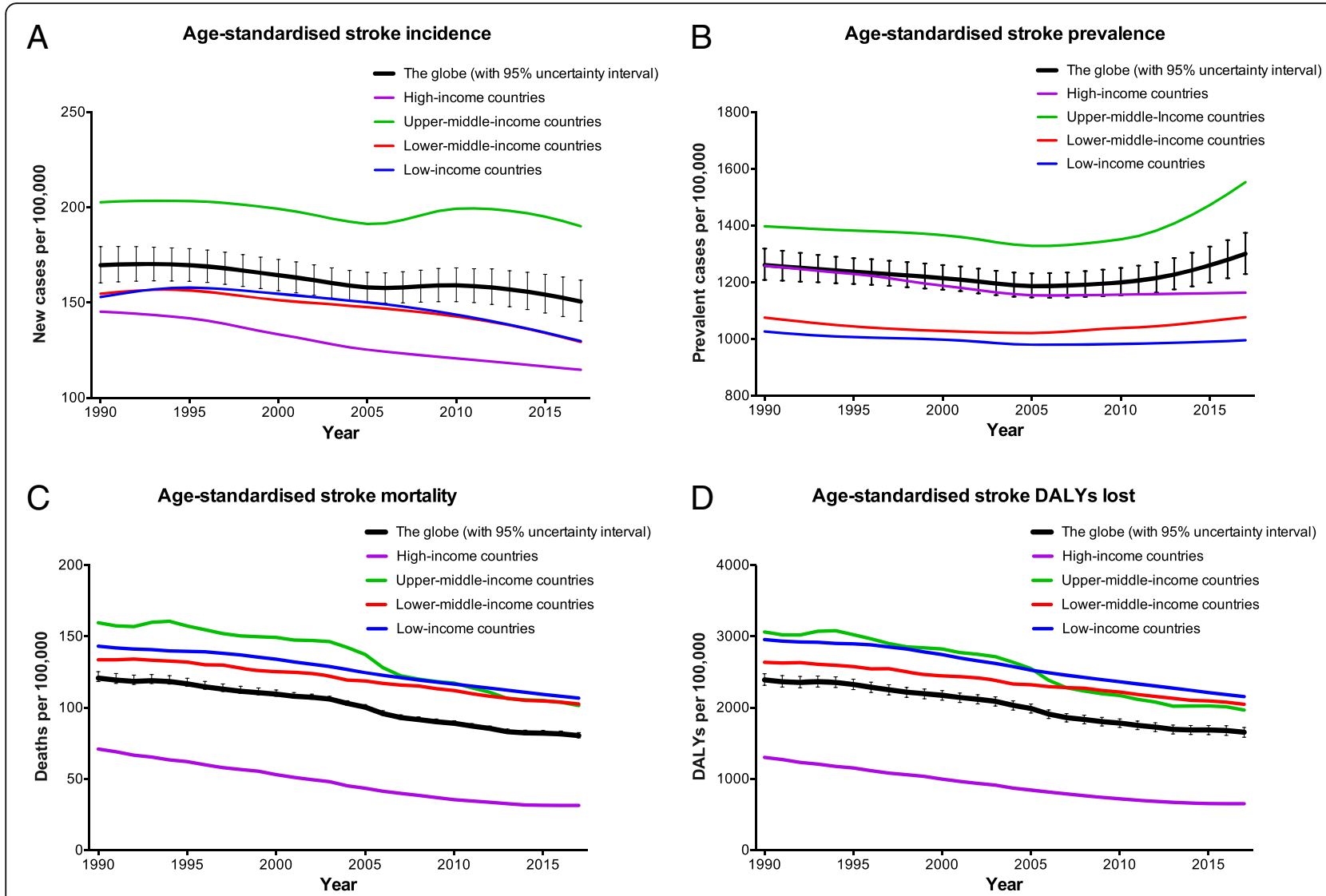

Fig. 1 Trends in age-standardised stroke $\mathbf{a}$ incidence, b prevalence, c mortality, and d disability-adjusted life years (DALYs) lost from 1990 to 2017 in different regions being classified as to the World Bank income levels (extracted from [8])

\section{Methods}

We extracted data from the Global Burden of Diseases, Injuries, and Risk Factors Study (GBD) 2017, coordinated by the Institute for Health Metrics and Evaluation, as of 19 May 2019 [8]. We reported global and SESspecific rates of age-standardised and age-specific stroke incidence, prevalence, mortality, and disability-adjusted life years (DALYs) lost per 100,000 population with 95\% uncertainty interval (UI) based on the 25th and 975th values of the ordered 1000 draws between 1990 and 2017. The method for estimating the parameters is explained elsewhere [9-11]. In summary, the GBD enterprise originated from the 1990 World Bank study that was commissioned to measure the status of global health. It comprises information from multiple sources including multiple databases such as MEDLINE, EMBASE, LILACS, Scopus, PubMed and Science Direct, Global Health Database, WHO library and WHO regional databases, VR systems, sample registration systems, household surveys (complete birth histories, summary birth histories, sibling histories), censuses (summary birth histories, household deaths), and Demographic Surveillance Sites (DSS). DisMod-MR 2.0 was used as a meta-regression tool to pool the case fatality data and generate location-year-age-sex-specific case fatality rate estimates.

SES was defined based on the gross national per capita income, as classified by the World Bank (i.e. low-income, lower-middle-income, upper-middle-income, or highincome) [3], and the Socio-Demographic Index (SDI; i.e. low-SDI, low-middle-SDI, middle-SDI, high-middle-SDI, or high-SDI) being developed in the GBD 2016. We also retrieved ranks of ten leading causes of death and strokerelated death attributable to common modifiable risk factors in those regions. We calculated rates and proportions of the abovementioned factors in both and each sexes of different socioeconomic regions from 1990 to 2017. We assessed proportions of the incidence, prevalence, burden, and mortality of stroke in different age groups. Unless explicitly mentioned otherwise, all rates were agestandardised using the GBD standard and reported per 100,000 population [12]. We used Numbers (version 3.6.2 for Mac OS X, Apple Inc., USA) for data compilation, preliminary analyses, and making tables. We used Prism (version $6.0 \mathrm{~h}$ for Mac OS X, GraphPad Software Inc.) for analysis and making the graphs. 
Thirty-one LICs include Afghanistan, Benin, Burkina Faso, Burundi, Central African Republic, Chad, Comoros, Democratic Republic of the Congo, Eritrea, Ethiopia, Guinea, Guinea-Bissau, Haiti, Liberia, Madagascar, Malawi, Mali, Mozambique, Nepal, Niger, North Korea, Rwanda, Senegal, Sierra Leone, Somalia, South Sudan, Tanzania, The Gambia, Togo, Uganda, and Zimbabwe; 52 LMICs include Angola, Armenia, Bangladesh, Bhutan, Bolivia, Cambodia, Cameroon, Cape Verde, Congo, Côte d'Ivoire, Djibouti, Egypt, El Salvador, Federated States of Micronesia, Georgia, Ghana, Guatemala, Honduras, India, Indonesia, Jordan, Kenya, Kiribati, Kyrgyzstan, Laos, Lesotho, Mauritania, Moldova, Mongolia, Morocco, Myanmar, Nicaragua, Nigeria, Pakistan, Palestine, Papua New Guinea, Philippines, Sao Tome and Principe, Solomon Islands, Sri Lanka, Sudan, Swaziland, Syria, Tajikistan, Timor-Leste, Tunisia, Ukraine, Uzbekistan, Vanuatu, Vietnam, Yemen, and Zambia; 54 UMICs include Albania, Algeria, American Samoa, Argentina, Azerbaijan, Belarus, Belize, Bosnia and Herzegovina, Botswana, Brazil, Bulgaria, China, Colombia, Costa Rica, Croatia, Cuba, Dominica, Dominican Republic, Ecuador, Equatorial Guinea, Fiji, Gabon, Grenada, Guyana, Iran, Iraq, Jamaica, Kazakhstan, Lebanon, Libya, Macedonia, Malaysia, Maldives, Marshall Islands, Mauritius, Mexico, Montenegro, Namibia, Panama, Paraguay, Peru, Romania, Russian Federation, Saint Lucia, Saint Vincent and the, Grenadines, Samoa, Serbia, South Africa, Suriname, Thailand, Tonga, Turkey, Turkmenistan, and Venezuela; and 58 HICs include Andorra, Antigua and Barbuda, Australia, Austria, Bahrain, Barbados, Belgium, Bermuda, Brunei, Canada, Chile, Cyprus, Czech Republic, Denmark, Estonia, Finland, France, Germany, Greece, Greenland, Guam, Hungary, Iceland, Ireland, Israel, Italy, Japan, Kuwait, Latvia, Lithuania, Luxembourg, Malta, Netherlands, New Zealand, Northern Mariana Islands, Norway, Oman, Poland, Portugal, Puerto Rico, Qatar, Saudi Arabia, Seychelles, Singapore, Slovakia, Slovenia, South Korea, Spain, Sweden, Switzerland, Taiwan, The Bahamas, Trinidad and Tobago, United Arab Emirates, the UK, Uruguay, Virgin Islands, and the USA.

\section{Results}

\section{Stroke incidence, prevalence, mortality, and burden}

Based on the GBD 2017 [8], the global crude number of new stroke events has increased by $76 \%$ (UI 71-80\%) from 6.8 (UI 6.4-7.2) million new events in 1990 to 11.9 (UI 11.1-12.8) million in 2017 (Table 2). However, the age-standardised global stroke incidence rate (i.e. new stroke events per 100,000 population) decreased overall by $11 \%$ (UI $9-12 \%$; $-15 \%$ in LICs, $-16 \%$ in LMICs, $-6 \%$ in UMICs, and $-21 \%$ in HICs) during the same period of time. The age-standardised global rate of new strokes became 150.5 (UI 140.3-161.8) per 100,000 in 2017. This decrease could be partly explained by more aggressive preventive measures and control of the risk factors. Nevertheless, despite a doubling of the global number of new ischaemic strokes from 1990 to 2017, there was no significant change in its age-standardised rate, while haemorrhagic events have significantly decreased globally and regionally during this period. Ischaemic strokes and intracerebral (not subarachnoid) haemorrhage seem to be more common in males than females; female to male ratio has decreased by 6\% from 1990 to 2017.

In contrast, stroke prevalence has increased over time from 1990 to 2017, likely because of longer survival and reduced mortality of people experiencing a stroke. In 2017, the crude number of people with a stroke was 104.2 million (UI 98.5-110.1), which has almost doubled, particularly for ischaemic stroke, compared to the number in 1990 (Table 2). The global rate of agestandardised stroke prevalence has increased by 3\% (UI $1-5 \%$ ) from 1990 to 2017 to reach to 1300.6 (UI $1229.0-1374.7)$ per 100,000 in 2017; particularly in UMICs (11\%, UI 8-14\%). This increase in prevalence could be partly explained by improved healthcare (including screening, prevention, diagnosis, and treatment) and general awareness, which has extended the lifespan of stroke patients in these income categories. Contrarily, both LICs and HICs have exhibited a respective 3\% (UI $1-5 \%$ ) and $8 \%$ (UI 5-10\%) decrease in the agestandardised rates of stroke prevalent cases by 2017. Of note, in contrast to ischaemic strokes, the agestandardised rates of haemorrhagic strokes have significantly decreased worldwide from 1990 to 2017.

Stroke is the second leading cause of death worldwide, with regard to the age-standardised global stroke mortality rate (i.e. stroke-related deaths per $100,000)$, and this rank has remained relatively constant in different regions since 1990 (Table 1). Because of population growth, particularly among the elderly, the crude number of stroke events and mortality has dramatically increased worldwide from 1990 to 2017. However, compared to 1990, the agestandardised global (ischaemic and haemorrhagic) stroke mortality rate has decreased by $33 \%$ (UI $32-$ 35 ; $-25 \%$ in LICs, $-23 \%$ in LMICs, $-36 \%$ in UMICs, and $-56 \%$ in HICs). There were 80.5 (UI 78.9-82.6) deaths per 100,000 in $2017,45 \%$ of which were related to ischaemic strokes (Table 2).

In 2017, stroke has imposed 132.1 (126.5 to 137.4) million DALYs lost globally (34\% more than in 1990), $42 \%$ of which was related to ischaemic strokes, in particular, 6.8 million DALYs in LICs, 47.1 million DALYs in LMICs, 63.1 million DALYs in UMICs, and 14.2 million DALYs in HICs (Table 2). Nevertheless, the agestandardised rate of DALYs lost has decreased globally by 31\% (UI 29-33), from 2392.7 (UI 2316.5-2478.9) in 1990 to $1657.2(1587.4-1723.8)$ in 2017. The decrease 


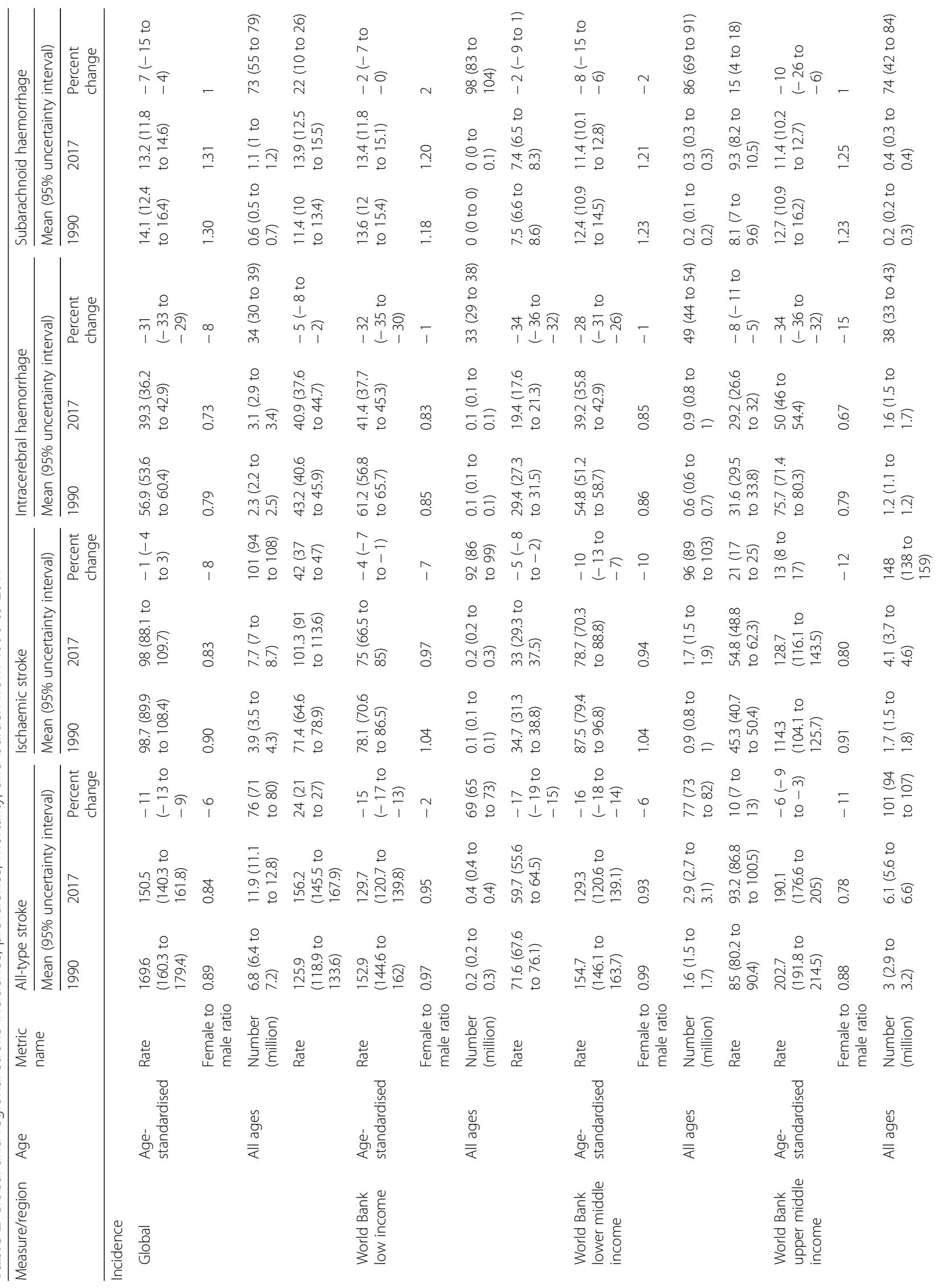




\begin{tabular}{|c|c|c|c|c|c|c|c|c|c|c|c|c|c|c|c|}
\hline 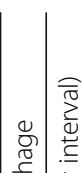 & 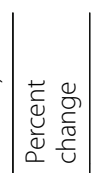 & 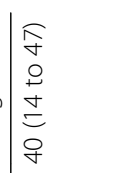 & $\begin{array}{l}a \\
0 \\
0 \\
\stackrel{d}{d} \\
i n\end{array}$ & 0 & $\begin{array}{l}\overline{8} \\
0 \\
0 \\
0 \\
6 \\
\overline{6}\end{array}$ & $\begin{array}{l}\bar{a} \\
\dot{m} \\
0 \\
\bar{m} \\
i n \\
m\end{array}$ & $\begin{array}{l}0 \\
0 \\
0 \\
1 \\
0 \\
0 \\
1 \\
1 \\
1\end{array}$ & 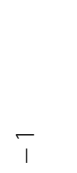 & $\begin{array}{l}0 \\
0 \\
0 \\
0 \\
\infty \\
\stackrel{0}{N} \\
N\end{array}$ & $\begin{array}{l}\stackrel{1}{1} \\
\stackrel{0}{o} \\
\stackrel{\Xi}{\Xi}\end{array}$ & $\begin{array}{l}\widehat{a} \\
0 \\
+ \\
+ \\
I \\
-1\end{array}$ & $\sim$ & 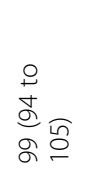 & v & $\begin{array}{l}\stackrel{0}{T} \\
\stackrel{1}{1} \\
0 \\
1\end{array}$ \\
\hline 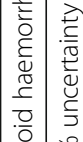 & $\hat{\bar{i}}$ & 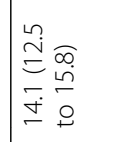 & 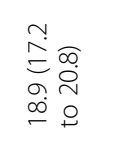 & $\underset{f}{+}$ & 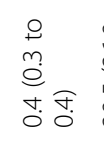 & 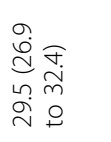 & 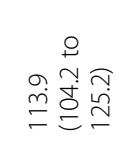 & $\stackrel{\text { If }}{\lessgtr}$ & 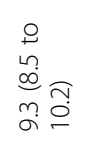 & 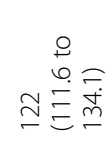 & 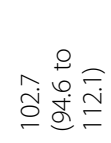 & $\stackrel{+}{m}$ & 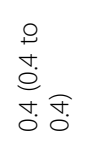 & $\begin{array}{l}6 \\
\text { o } \\
0 \\
0\end{array}$ & 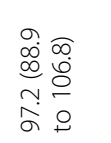 \\
\hline 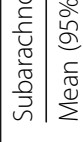 & \& & 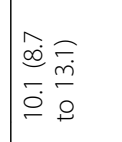 & 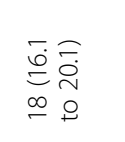 & 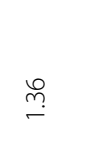 & 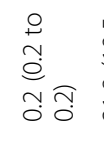 & 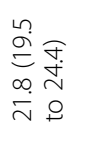 & 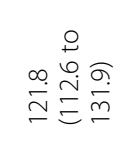 & $\stackrel{\infty}{+}$ & 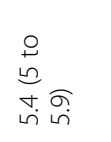 & 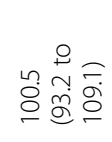 & 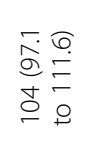 & $\stackrel{\sim}{m}$ & 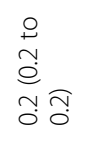 & o. & 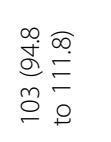 \\
\hline ב. & 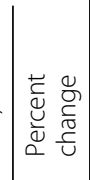 & $\begin{array}{l}\frac{1}{n} \\
0 \\
0 \\
0 \\
0 \\
0\end{array}$ & 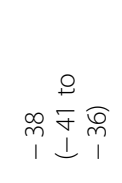 & † & 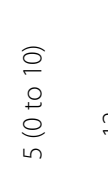 & 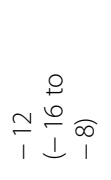 & 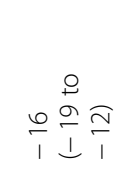 & $i$ & $\begin{array}{l}\infty \\
\stackrel{\infty}{0} \\
0 \\
0\end{array}$ & $\begin{array}{l}\bar{E} \\
\pm \\
=\end{array}$ & 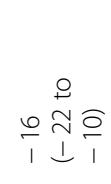 & $\circ$ & $\begin{array}{l}\bar{c} \\
\infty \\
0 \\
\infty \\
0 \\
0 \\
2\end{array}$ & 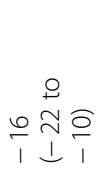 & $=\stackrel{\overrightarrow{0}}{=} \underset{1}{\tau}$ \\
\hline 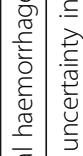 & $\bar{i}$ & $\begin{array}{l}0 \\
0 \\
0 \\
0 \\
0 \\
0 \\
0 \\
0 \\
0 \\
0 \\
0\end{array}$ & 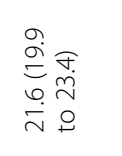 & $\underset{0}{\mathbb{O}}$ & 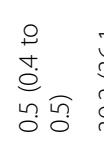 & 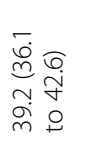 & 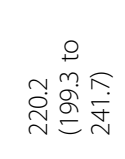 & $\stackrel{8}{-}$ & 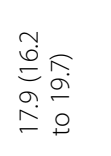 & 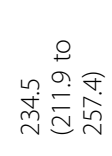 & 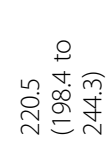 & $\stackrel{\Delta}{\circ}$ & $\begin{array}{l}0 \\
0 \\
\infty \\
\stackrel{0}{0} \\
\stackrel{0}{0}=\end{array}$ & 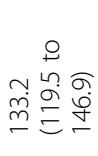 & 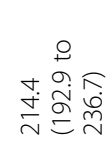 \\
\hline 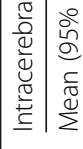 & O & 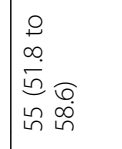 & 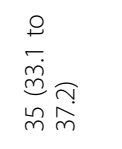 & $\stackrel{n}{\delta}$ & 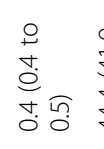 & 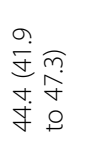 & 站 & $\stackrel{0}{\circ}$ & 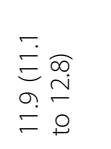 & 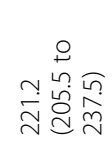 & 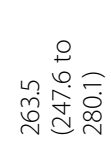 & $\stackrel{n}{o}$ & 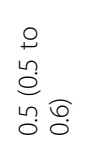 & 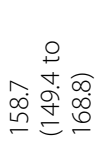 & 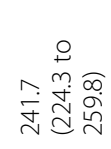 \\
\hline 㺼 & 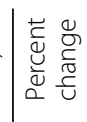 & 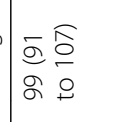 & 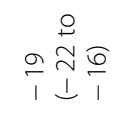 & 0 & 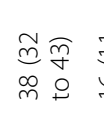 & 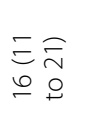 & $\begin{array}{l}\stackrel{0}{ \pm} \\
\stackrel{0}{\circ}\end{array}$ & $\stackrel{n}{p}$ & $\stackrel{\stackrel{P}{8}}{\cong} \underset{E}{E}$ & 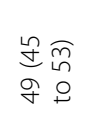 & $\stackrel{0}{0}$ & $\hat{\imath}$ & $\begin{array}{l}\hat{0} \widehat{0} \overline{0} \\
\circ \stackrel{0}{\circ}\end{array}$ & $\begin{array}{l}m \\
1 \\
-0 \\
1 \\
0\end{array}$ & 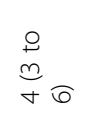 \\
\hline 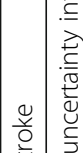 & $\bar{i}$ & 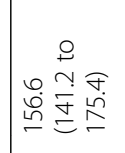 & 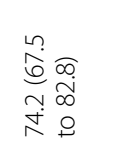 & 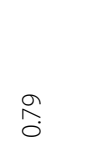 & 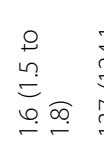 & 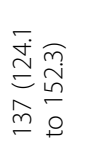 & 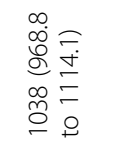 & ㅇ. & 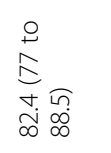 & 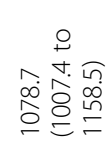 & 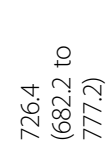 & 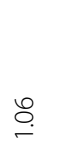 & 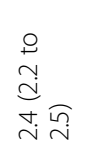 & 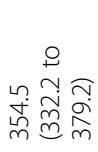 & $\begin{array}{c}\overbrace{0} \\
\infty\end{array}$ \\
\hline 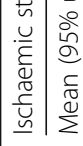 & S & 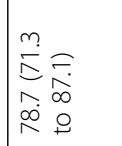 & 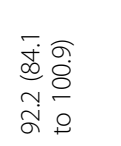 & 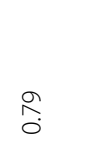 & 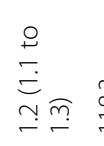 & 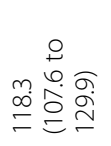 & 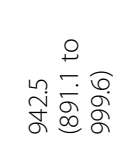 & ํ. & 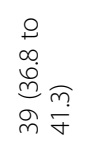 & 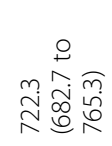 & 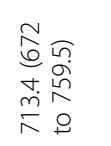 & $\stackrel{\Xi}{\check{E}}$ & 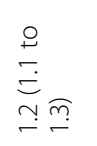 & 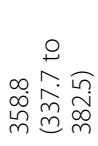 & รุ \\
\hline & 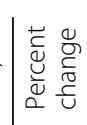 & 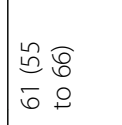 & 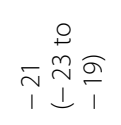 & ナ & 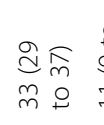 & $\begin{array}{l}\stackrel{0}{+} \\
\stackrel{9}{\sigma} \\
=\stackrel{\Omega}{n}\end{array}$ & $\begin{array}{l}\stackrel{P}{=} \\
m \sqrt{n}\end{array}$ & $\varphi$ & 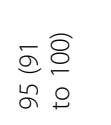 & 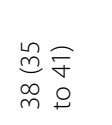 & $\begin{array}{ll}1 & 1 \\
m & 1 \\
1 & 0\end{array}$ & in & 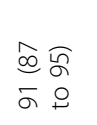 & & $\begin{array}{l}1 \approx \\
1 \\
0 \\
0\end{array}$ \\
\hline है & $\overline{\grave{D}}$ & 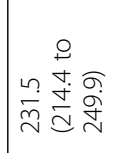 & 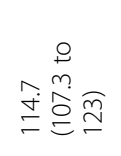 & $\stackrel{\infty}{\infty}$ & $\begin{array}{l}\stackrel{o}{+} \\
\stackrel{n}{d} \\
\stackrel{v}{\sim} \underset{\sim}{\sim}\end{array}$ & 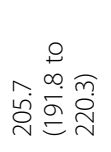 & 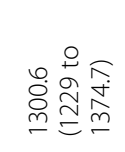 & ஃ̊ & 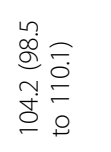 & 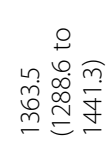 & 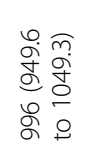 & $\stackrel{\infty}{\stackrel{\infty}{\sim}}$ & $\begin{array}{l}\stackrel{P}{N} \\
\stackrel{n}{m} \\
\stackrel{m}{m} \sqrt[n]{n}\end{array}$ & 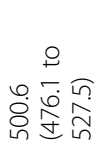 & $\begin{array}{l}\infty \\
0 \\
0 \\
0 \\
0 \\
0\end{array}$ \\
\hline 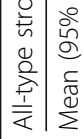 & 8 & 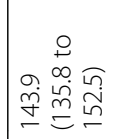 & 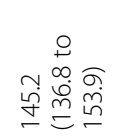 & $\underset{\infty}{\infty}$ & 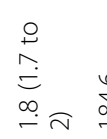 & 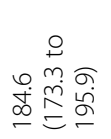 & 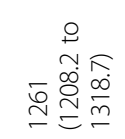 & & 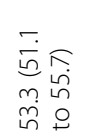 & 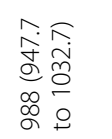 & 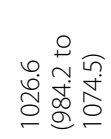 & & 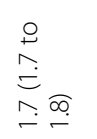 & 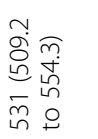 & 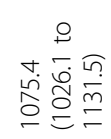 \\
\hline 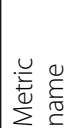 & & 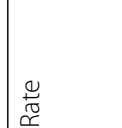 & $\underset{\pi}{\frac{\pi}{\pi}}$ & 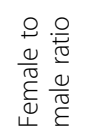 & 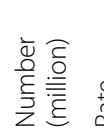 & & & 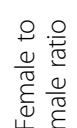 & 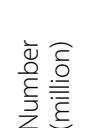 & $\underset{\pi / \pi}{\mathbb{N}}$ & & 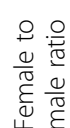 & 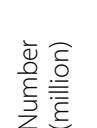 & $d$ & \\
\hline 岁 & & & 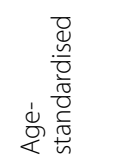 & & $\begin{array}{l}\stackrel{\widetilde{\varpi}}{D} \\
\bar{\Xi}\end{array}$ & & 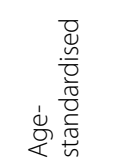 & & $\underset{\widetilde{\varpi}}{\stackrel{\widetilde{\sigma}}{\sigma}}$ & & 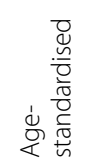 & & ్ֶర & & 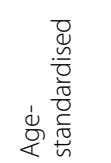 \\
\hline c. & & & 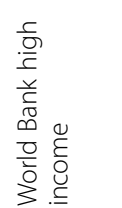 & & & & $\mathscr{Z}_{2}^{2}$ & & & & 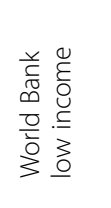 & & & & 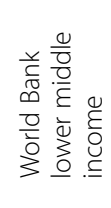 \\
\hline
\end{tabular}




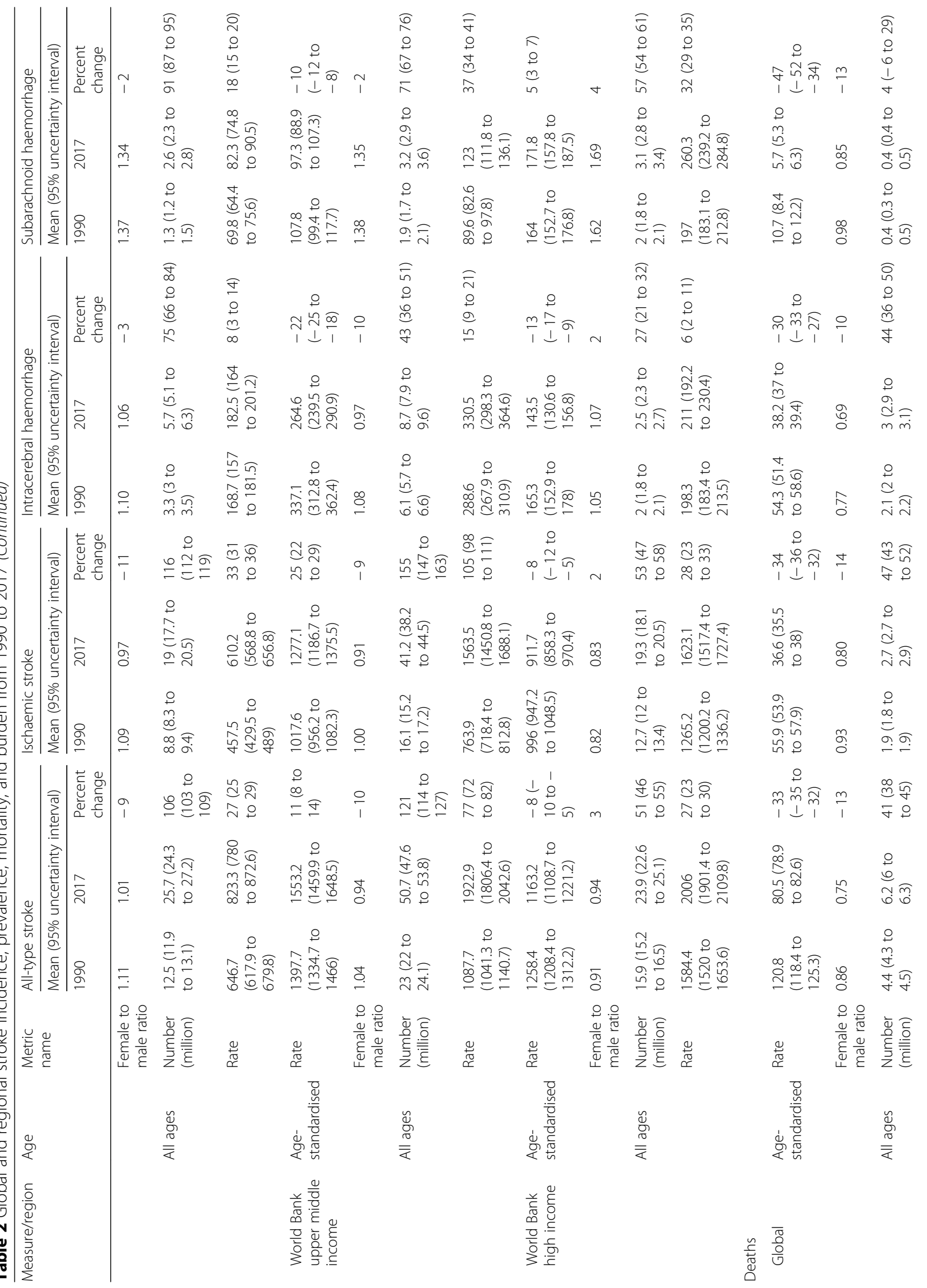




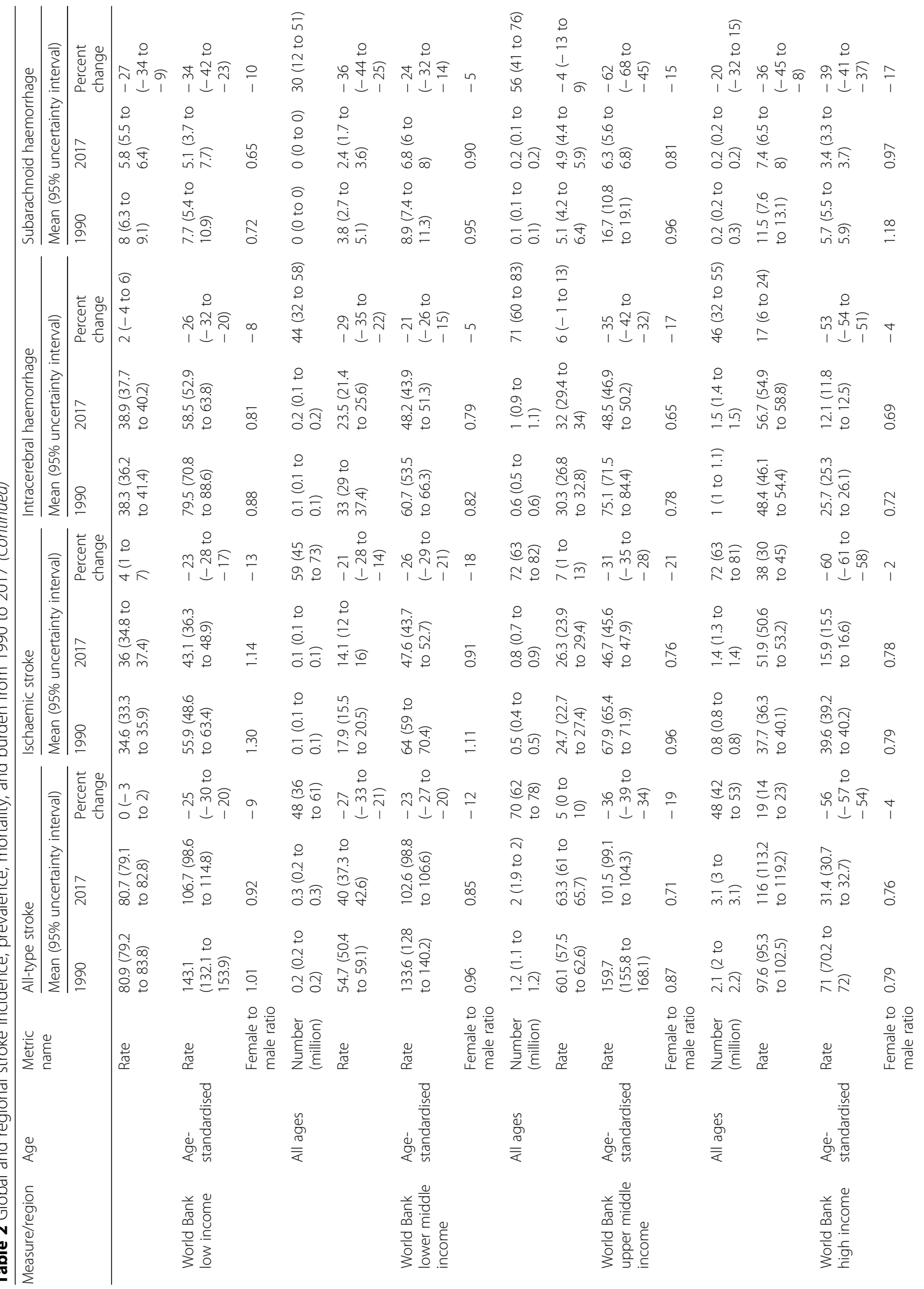




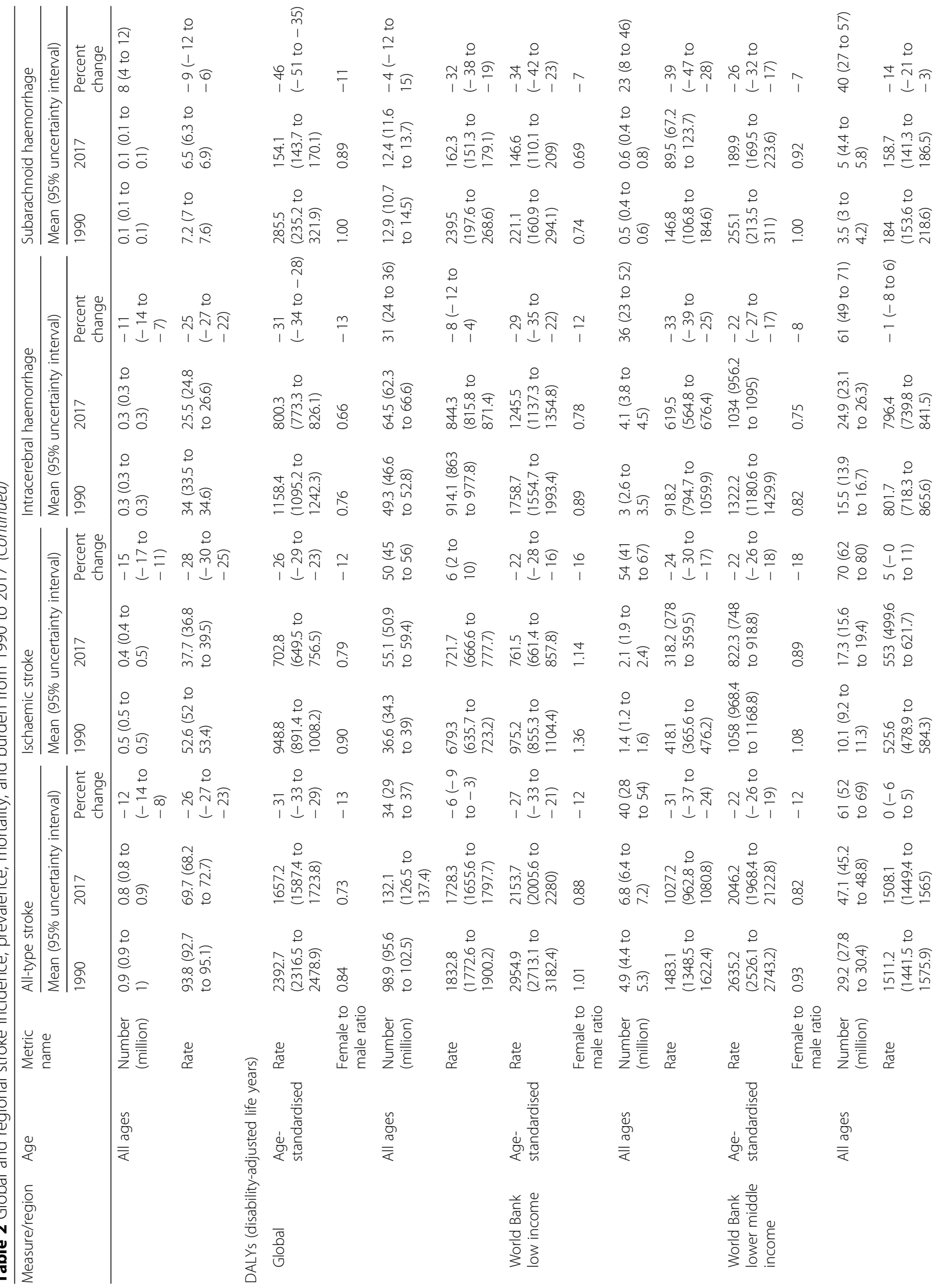




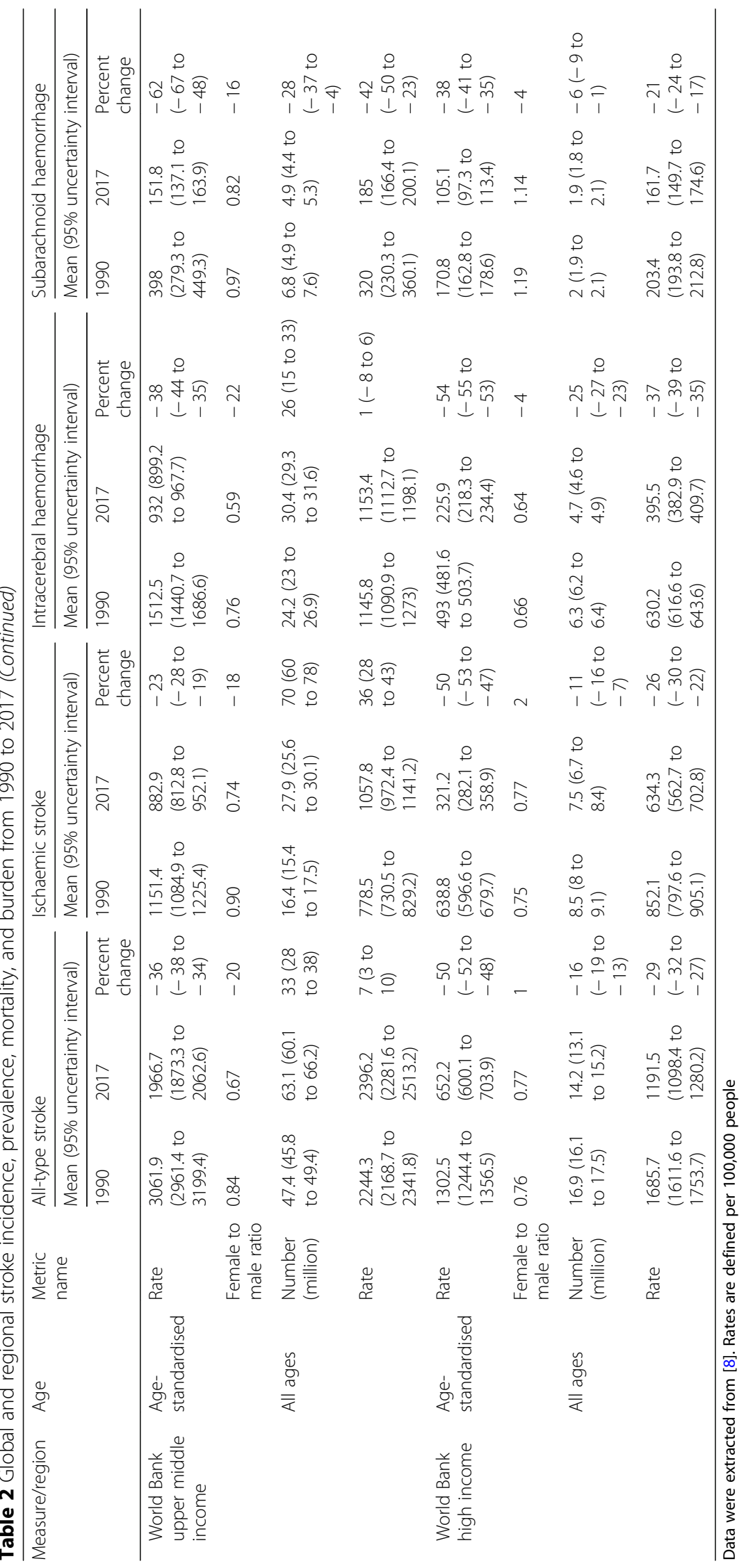


was more prominent in HICs and UMICs. The highest age-standardised rate of DALYs lost in 2017 for ischaemic stroke was in UMICs (882.9, UI 812.8-952.1); for intracerebral haemorrhage, the highest rate was in LICs (1245.5, UI1137.3-1354.8); and for subarachnoid haemorrhage the highest rate was in LMICs (189.9, UI 169.5223.6). Female to male ratio of stroke-related global DALYs lost was 0.73 in 2017, which has decreased by 13\% compared to that of 1990.

Overall, comparing the most-affected and the leastaffected income regions based on the age-standardised rates, there is a 1.7 -fold difference in stroke events (ranging from 190.1 [UI 176.6-205] per 100,000 in UMICs to 114.7 [UI 107.3-123] per 100,000 in HICs); a 1.6-fold difference in stroke prevalent cases (ranging from 1553.2 [UI 1459.9-1648.5] per 100,000 in LICs to 996.0 [UI 949.6$1049.3]$ per 100,000 in UMICs); a 3.4 -fold difference in stroke-related deaths (ranging from 106.7 [UI 98.6-114.8] per 100,000 in LICs to 31.4 [UI 30.7-32.7] per 100,000 in HICs); and a 3.3-fold difference in stroke DALYs (ranging from 2153.7 [UI 2005.6-2280] per 100,000 in LICs to 652.2 [UI 600.1-703.9] per 100,000 in HICs).

\section{Modifiable predictors of stroke mortality}

In 2017, 5.2 million stroke-related deaths and 116.3 million stroke-related DALYs lost worldwide were attributable to modifiable risk factors, less than half of which were observed for ischaemic strokes (Additional file 2: Table S1). Since 1990, the mean age-standardised global (ischaemic and haemorrhagic) stroke mortality rates attributable to modifiable risk factors have declined by $34 \%$ (UI $30-37 \%$ ), ranging from $23 \%$ in LMICs to $58 \%$ in HICs (Table 3 and Additional file 4: Table S3). The agestandardised rate of global stroke deaths per 100,000 attributable to modifiable risk factors was 67.9 (UI 64.2$71.3)$ per 100,000 in 2017 . This could be explained by improvements in quality of life and many SES determinants, particularly infrastructures, healthcare, and general awareness. However, some unhealthy habits of modern life have inevitably resulted in an increased stroke mortality rates attributable to some of the underlying risk factors, such as smoking, obesity, and alcohol drinking in less wealthy societies. Overall, there is a twofold to fivefold difference in stroke mortality rates attributable to modifiable risk factors between the most-affected and the least-affected SESspecific countries. Common predictors of stroke risk and mortality are discussed in the following and in Additional file 1: Text S1.

\section{Hypertension}

Globally, hypertension has remained the leading modifiable predictor of stroke mortality since 1990 irrespective of SES (Table 4). This is despite a 32.7\% decrease in hypertensionattributed risk of age-standardised global stroke mortality rate from 1990 to 2017, which varied from 19.7\% decrease in LICs to $61.7 \%$ decrease in HICs (Table 3). It has also remained the top predictor of stroke mortality in different countries being classified based on SDI (Table 4). In 2017, deaths related to ischaemic rather than haemorrhagic strokes attributable to high systolic blood pressure were more common among women than men (Additional file 3: Table S2). Overall, there is a 3.8-fold difference in rates of stroke mortality attributable to hypertension between the most-affected and the least-affected income category, from 59.1 per 100,000 in LICs to 15.4 per 100,000 in HICs.

\section{Dietary risks}

Poor dietary habits (i.e. a diet low in fibre, fruits, vegetables, legumes, whole grains, nuts and seeds, milk, calcium, or seafood, and high in red meat, eggs, processed meat, sugar-sweetened beverages, trans-fatty acids, or sodium) are globally the second leading cause of stroke mortality, irrespective of income levels or SDI (Table 4). However, from 1990 to 2017, there was a 36.7\% decrease in dietaryattributed risk of age-standardised global stroke mortality rate, which varied from $28.7 \%$ decrease in LICs to $57.3 \%$ decrease in HICs (Table 3). Overall, there is a 4.3-fold difference in rates of stroke mortality attributable to dietary risks between the most-affected and the least-affected income category, from 49.2 per 100,000 in LICs to 11.4 per 100,000 in HICs.

In addition, dietary risks can worsen the consequences of stroke. In particular, diets low in fruits, low in whole grains, low in vegetables, high in sodium, and high in sugar-sweetened beverages increase the likelihood of global stroke mortality (Fig. 2). Worldwide, there is a downward trend in stroke events attributable to dietary risks in different SES regions (Fig. 2). The downslope became steeper in the UMICs since 2005, particularly in European and Western Pacific regions compared to the others. This suggests that better education on healthy diets is needed in these areas. It is also possible that the speed of deterioration in other cofactors may counteract the improvement in dietary risks.

\section{Diabetes mellitus}

Diabetes and glucose intolerance-related mortality is globally the third critical risk factor of stroke mortality in 2017 (Table 4). However, the age-standardised global stroke mortality rate attributable to diabetes mellitus has decreased by $25.0 \%$ from 1990 to 2017, which varied from $4.3 \%$ decrease in LMICs to $48.2 \%$ decrease in HICs (Table 3). Overall, there is a 3.5 -fold difference in rates of stroke mortality attributable to diabetes mellitus between the most-affected and the least-affected income category, from 27.1 per 100,000 in LICs to 7.8 per 100,000 in HICs. 


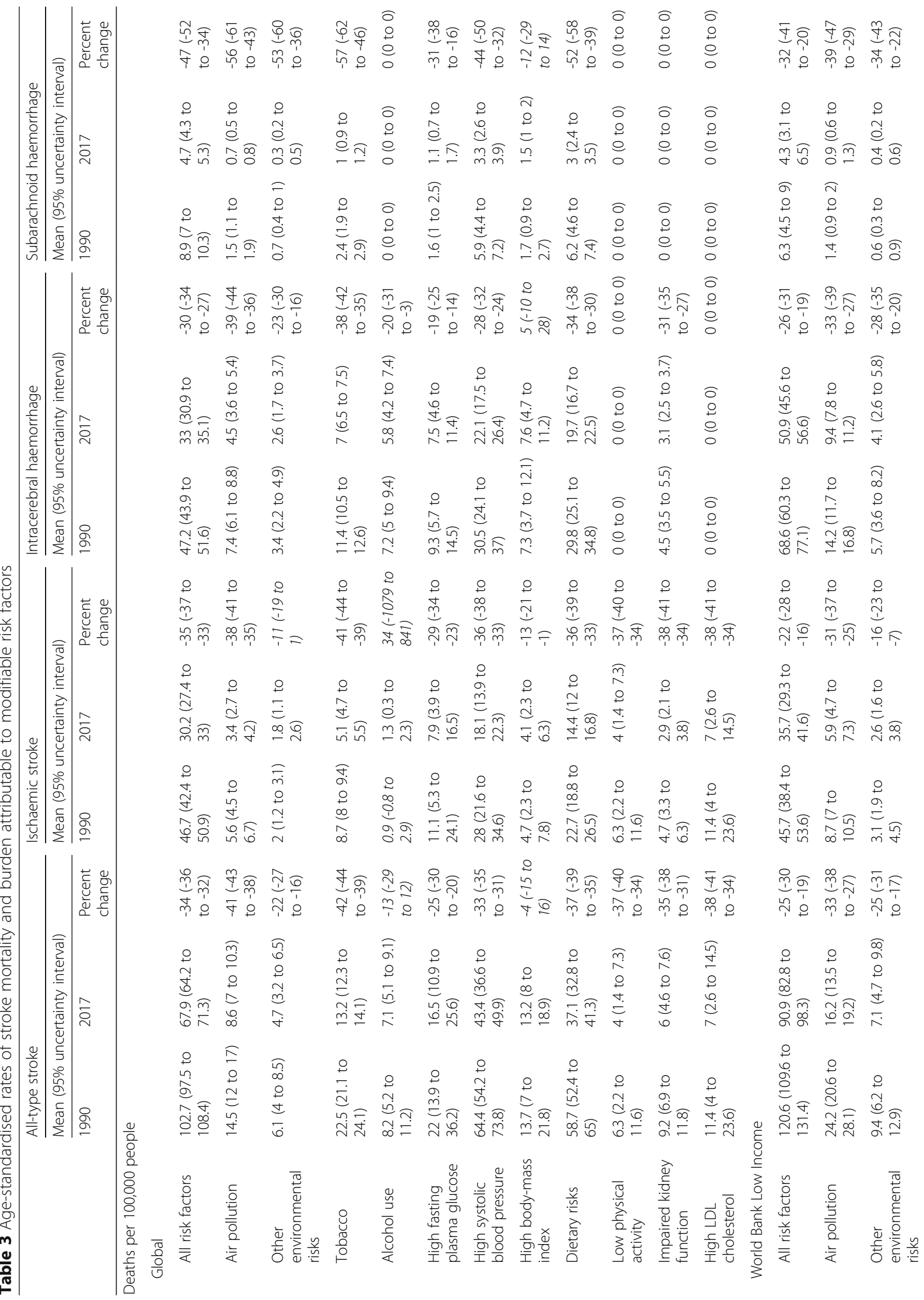




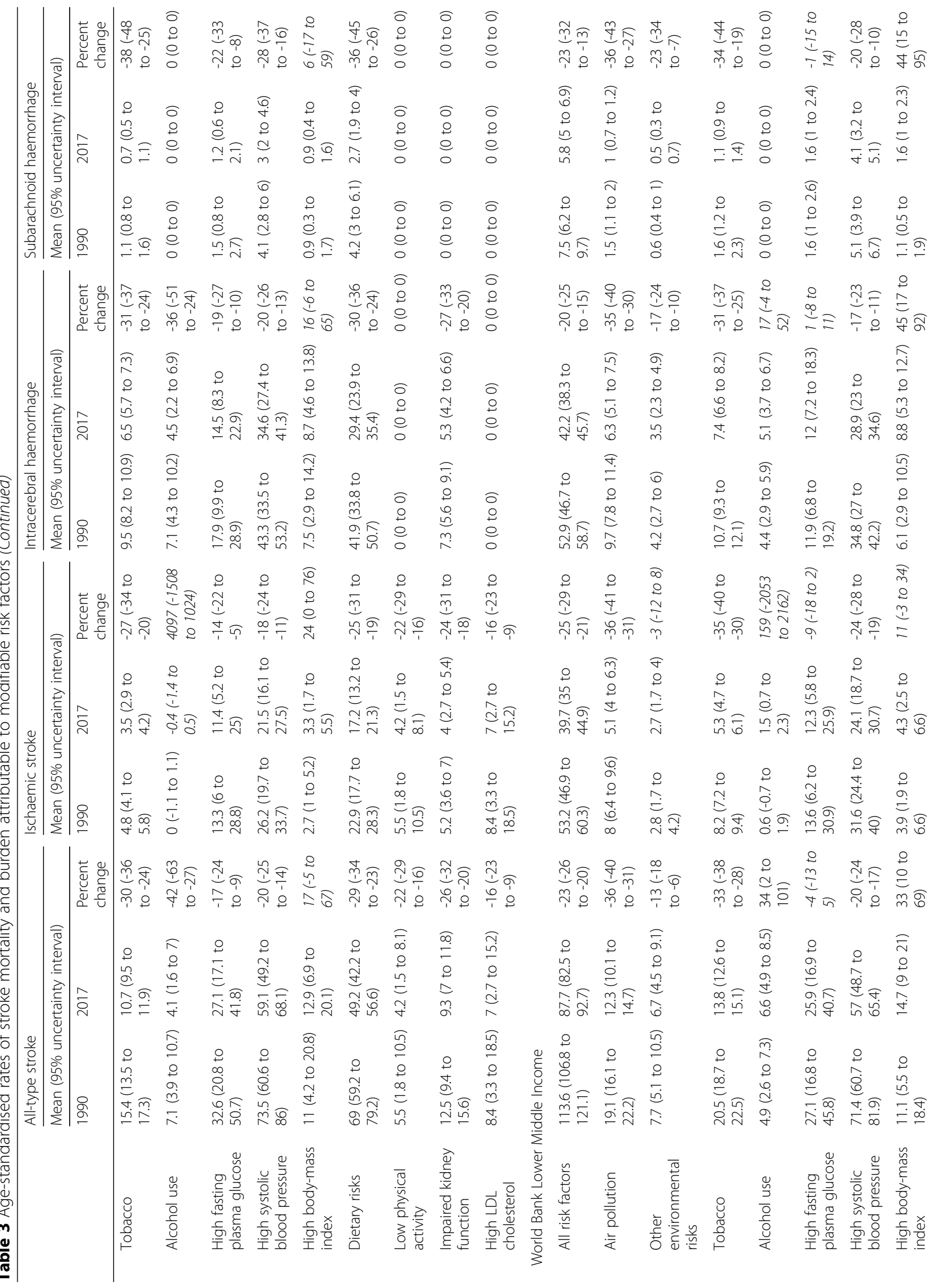




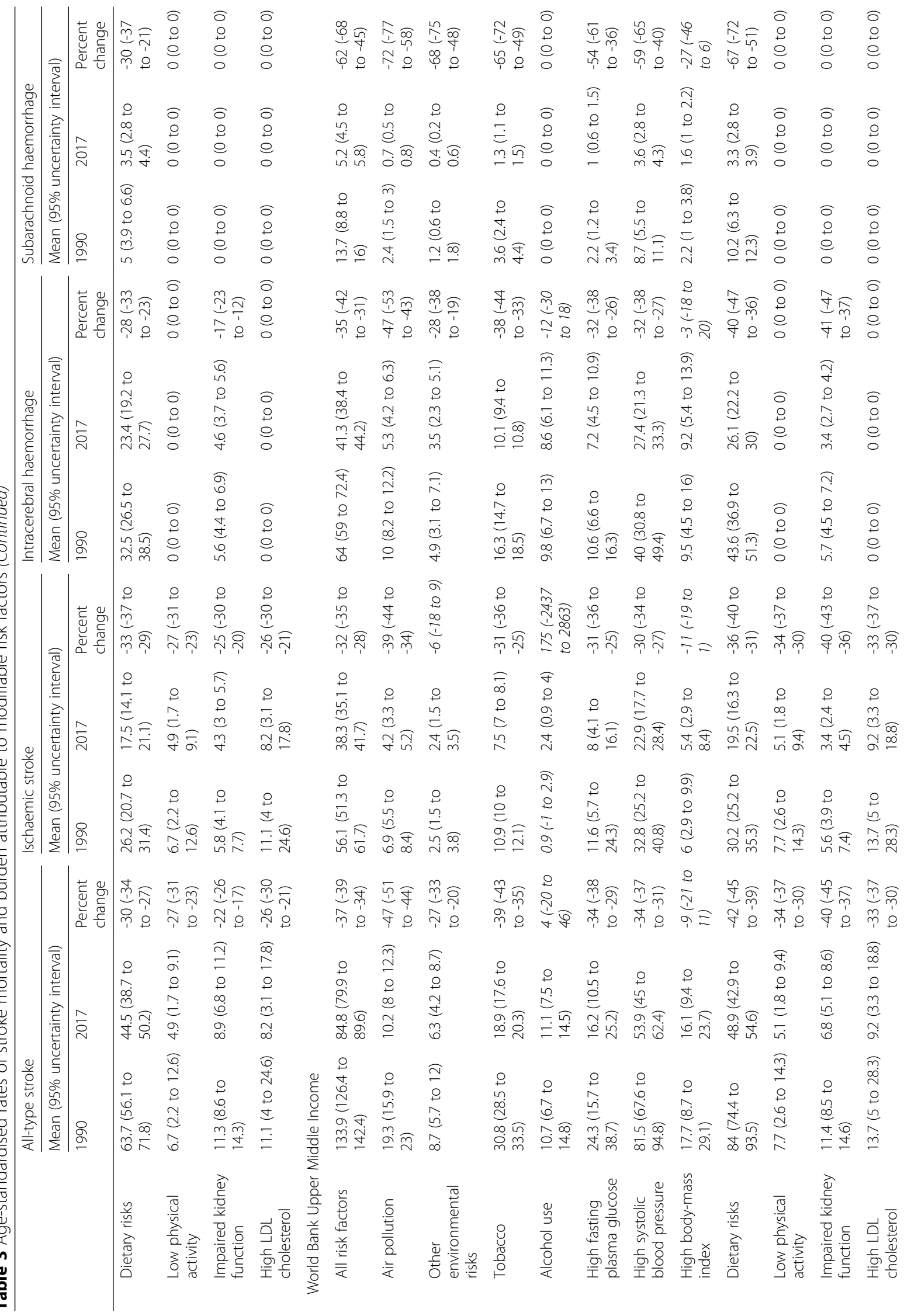




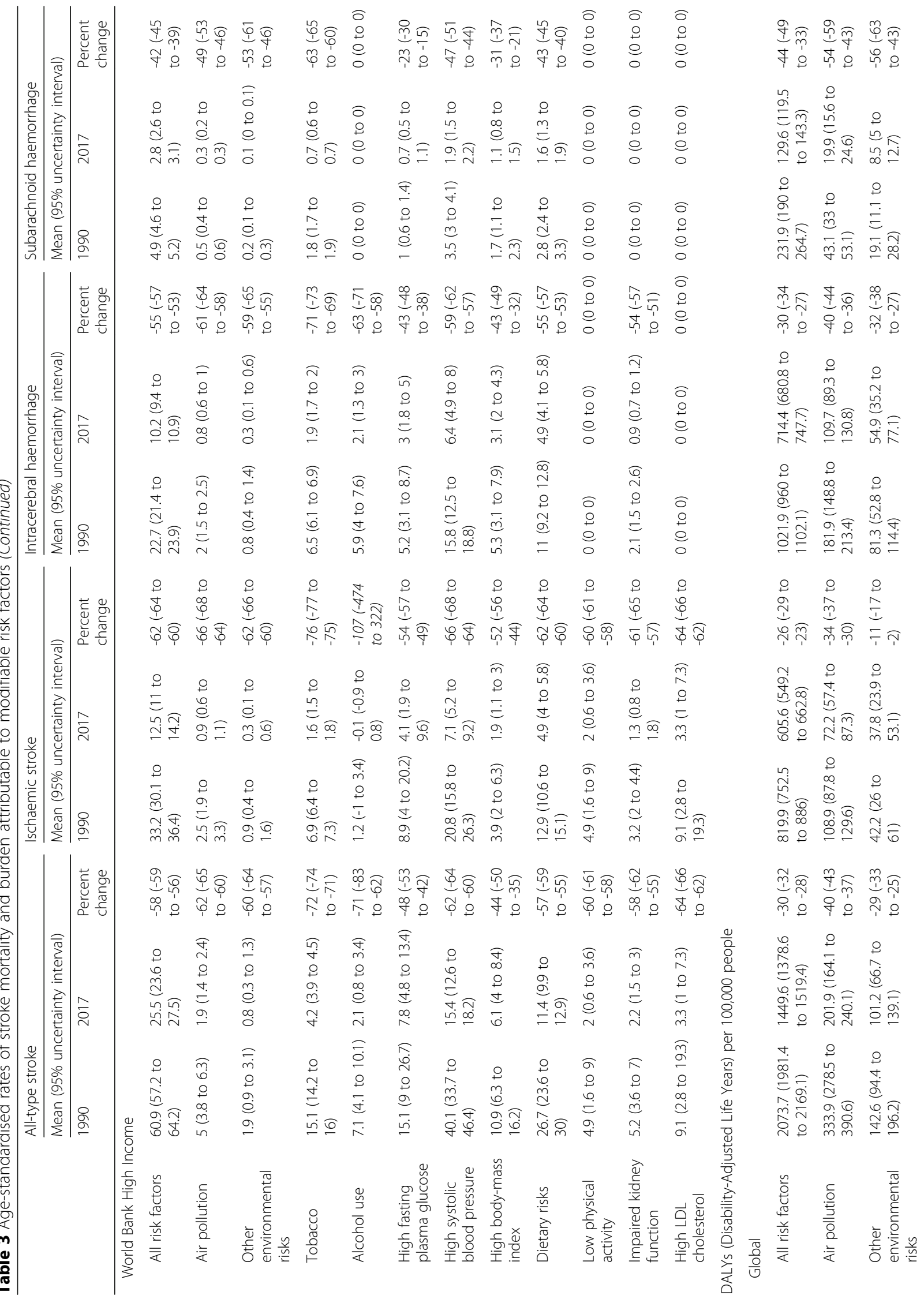




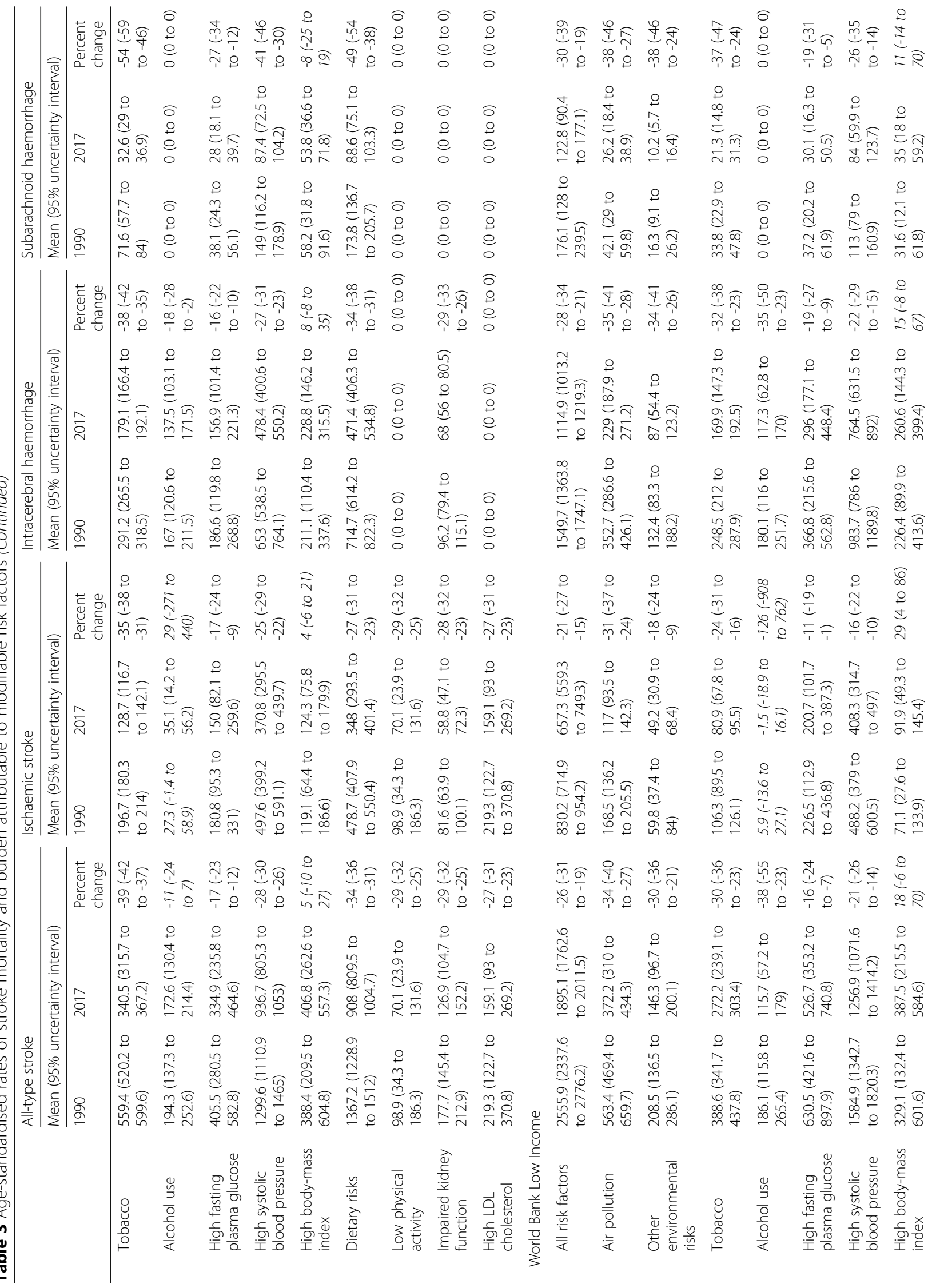




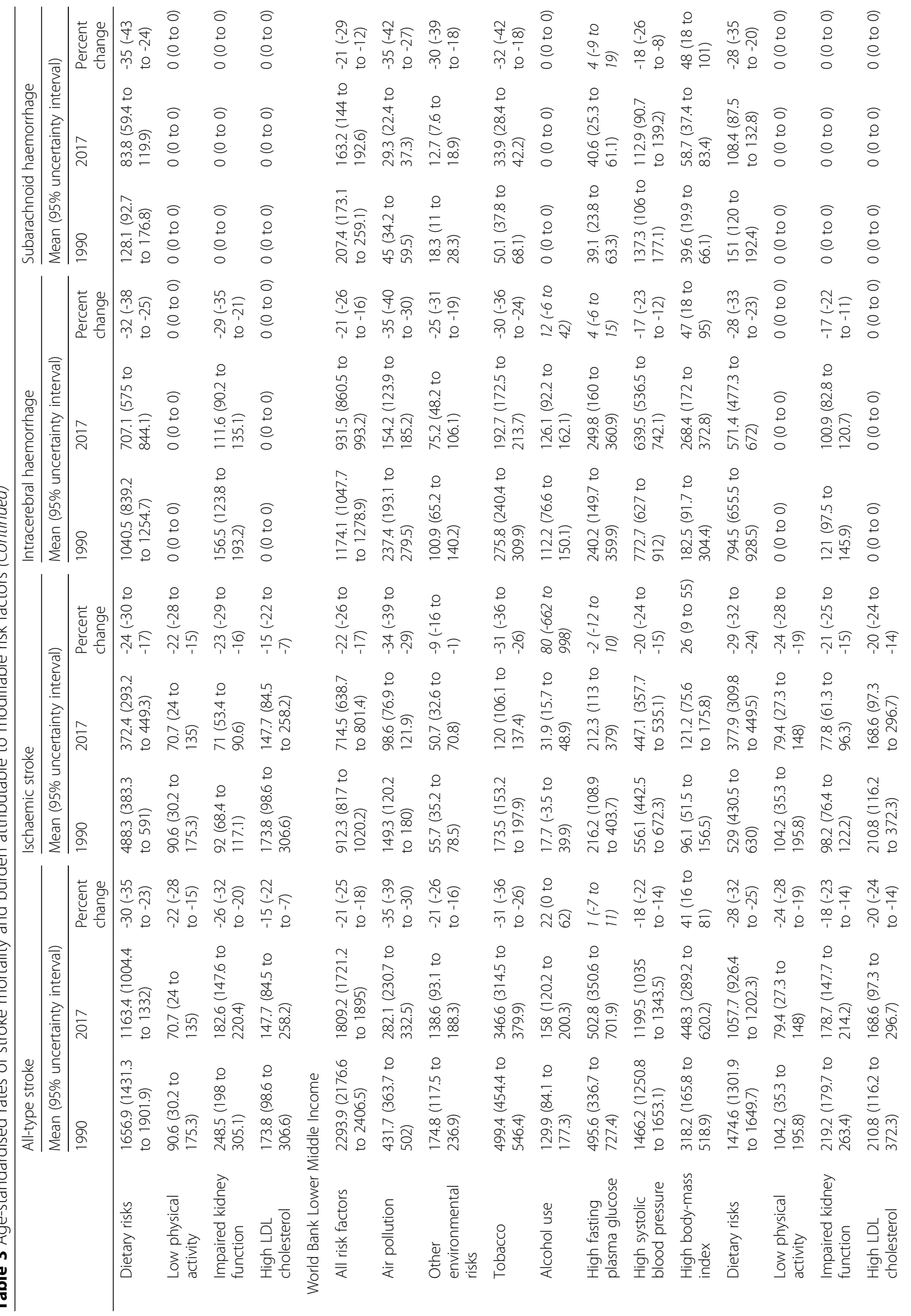




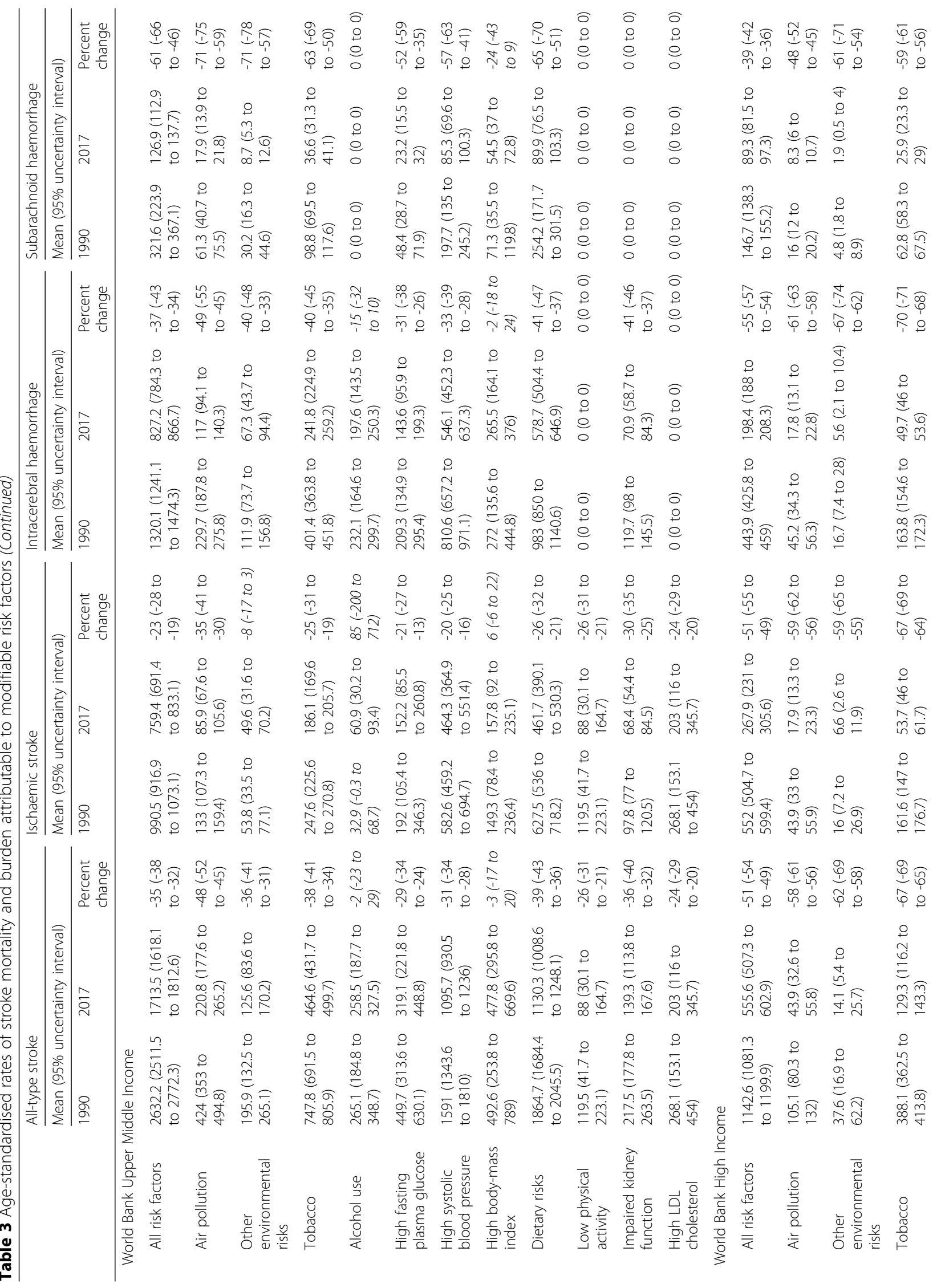




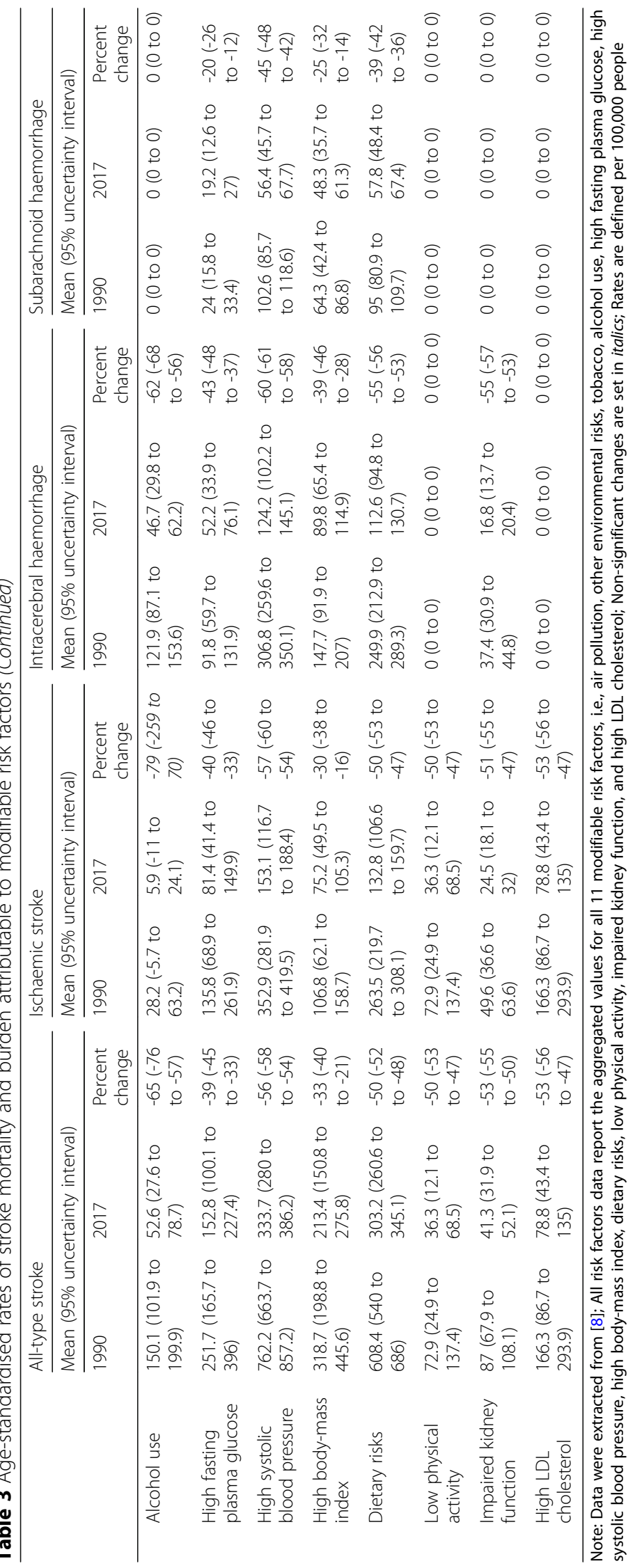


Table 4 Rank of age-standardised stroke mortality rate attributable to modifiable risk factors in (A) The World Bank (WB) low-income countries (LICS), lower-middle-income countries (LMICs), upper-middle-income countries (UMICs), and high-income countries (HICs) in 1990 and 2017 and (B) in different socioeconomic status regions classified by Socio-Demographic Index (SDI) in 1990 and 2017 (extracted from [8])

\begin{tabular}{|c|c|c|c|c|c|c|c|c|c|c|}
\hline \multirow{3}{*}{$\mathbf{A}$} & \multicolumn{10}{|c|}{$\begin{array}{l}\text { Age-standardised stroke mortality attributed to modifiable risk factors } \\
\text { (death rate per 100,000) }\end{array}$} \\
\hline & \multicolumn{2}{|c|}{ Global } & \multicolumn{2}{|c|}{ WB LICs } & \multicolumn{2}{|c|}{ WB LMICs } & \multicolumn{2}{|c|}{ WB UMICs } & \multicolumn{2}{|c|}{ WB HICs } \\
\hline & $\begin{array}{l}1990 \\
\text { rank }\end{array}$ & $\begin{array}{l}2017 \\
\text { rank }\end{array}$ & $\begin{array}{l}1990 \\
\text { rank }\end{array}$ & $\begin{array}{l}2017 \\
\text { rank }\end{array}$ & $\begin{array}{l}1990 \\
\text { rank }\end{array}$ & $\begin{array}{l}2017 \\
\text { rank }\end{array}$ & $\begin{array}{l}1990 \\
\text { rank }\end{array}$ & $\begin{array}{l}2017 \\
\text { rank }\end{array}$ & $\begin{array}{l}1990 \\
\text { rank }\end{array}$ & $\begin{array}{l}2017 \\
\text { rank }\end{array}$ \\
\hline Hypertension & 1 & 1 & 1 & 1 & 1 & 1 & 2 & 1 & 1 & 1 \\
\hline Dietary risks & 2 & 2 & 2 & 2 & 2 & 2 & 1 & 2 & 2 & 2 \\
\hline Tobacco & 3 & 5 & 5 & 6 & 4 & 5 & 3 & 3 & 4 & 5 \\
\hline Impaired glucose tolerance & 4 & 3 & 3 & 3 & 3 & 3 & 4 & 4 & 3 & 3 \\
\hline Air pollution & 5 & 6 & 4 & 4 & 5 & 6 & 5 & 7 & 8 & 9 \\
\hline Obesity & 6 & 4 & 6 & 5 & 7 & 4 & 6 & 5 & 5 & 4 \\
\hline Hypercholesterolemia & 7 & 8 & 7 & 7 & 6 & 7 & 7 & 8 & 6 & 6 \\
\hline Alcohol use & 8 & 7 & 8 & 9 & 9 & 8 & 8 & 6 & 7 & 7 \\
\hline Low physical activity & 9 & 9 & 9 & 8 & 8 & 9 & 9 & 9 & 8 & 8 \\
\hline \multirow[t]{2}{*}{ B } & \multicolumn{2}{|c|}{ Low SDI } & \multicolumn{2}{|c|}{$\begin{array}{l}\text { Low-middle } \\
\text { SDI }\end{array}$} & \multicolumn{2}{|c|}{ Middle SDI } & \multicolumn{2}{|c|}{$\begin{array}{l}\text { High-middle } \\
\text { SDI }\end{array}$} & \multicolumn{2}{|c|}{ High SDI } \\
\hline & $\begin{array}{l}1990 \\
\text { rank }\end{array}$ & $\begin{array}{l}2017 \\
\text { rank }\end{array}$ & $\begin{array}{l}1990 \\
\text { rank }\end{array}$ & $\begin{array}{l}2017 \\
\text { rank }\end{array}$ & $\begin{array}{l}1990 \\
\text { rank }\end{array}$ & $\begin{array}{l}2017 \\
\text { rank }\end{array}$ & $\begin{array}{l}1990 \\
\text { rank }\end{array}$ & $\begin{array}{l}2017 \\
\text { rank }\end{array}$ & $\begin{array}{l}1990 \\
\text { rank }\end{array}$ & $\begin{array}{l}2017 \\
\text { rank }\end{array}$ \\
\hline Hypertension & 1 & 1 & 1 & 1 & 2 & 1 & 1 & 1 & 1 & 1 \\
\hline Dietary risks & 2 & 2 & 2 & 2 & 1 & 2 & 2 & 2 & 2 & 2 \\
\hline Tobacco & 5 & 5 & 4 & 5 & 3 & 4 & 3 & 4 & 3 & 5 \\
\hline Impaired glucose tolerance & 3 & 3 & 3 & 3 & 4 & 3 & 4 & 3 & 4 & 3 \\
\hline Air pollution & 4 & 4 & 5 & 6 & 5 & 6 & 7 & 8 & 8 & 9 \\
\hline Obesity & 7 & 6 & 6 & 4 & 6 & 5 & 5 & 5 & 5 & 4 \\
\hline Hypercholesterolemia & 6 & 7 & 7 & 7 & 7 & 8 & 6 & 6 & 6 & 6 \\
\hline Alcohol use & 9 & 9 & 8 & 8 & 8 & 7 & 8 & 7 & 7 & 7 \\
\hline Low physical activity & 8 & 8 & 9 & 9 & 9 & 9 & 9 & 9 & 9 & 8 \\
\hline
\end{tabular}

Note: The order of risk factors has remained constant for an easier comparison between different socio-economical status regions

HICs high-income countries, LICS low-income countries, LMICS lower-middle-income countries, UMICS upper-middle-income countries, SDI Socio-Demographic Index, WB The World Bank

\section{Obesity}

Obesity is globally the fourth most influential indicator of stroke mortality, which varies slightly between different SES (Table 4). Although the age-standardised global stroke mortality rate attributable to obesity has decreased by $3.8 \%$, although non-significantly from 1990 to 2017, more prominently in HICs (44.0\%), its risk increased in both LICs and LMICs by $17.1 \%$ (statistically not significantly) and $32.7 \%$, respectively (Table 3 ). This is contrary to the higher proportion of obesity observed in HICs than
LMICs [13], which might be related to the occurrence of first stroke at younger ages in countries of lower-income levels (Table 5). The obesity epidemic is just more recent in middle-income countries as compared to HICs, given the ongoing epidemiological and nutritional transition happening in LMICs. Overall, there is a 2.6-fold difference in rates of stroke mortality attributable to obesity between the most-affected and the least-affected income category, from 16.1 per 100,000 in UMICs to 6.1 per 100,000 in HICs. 


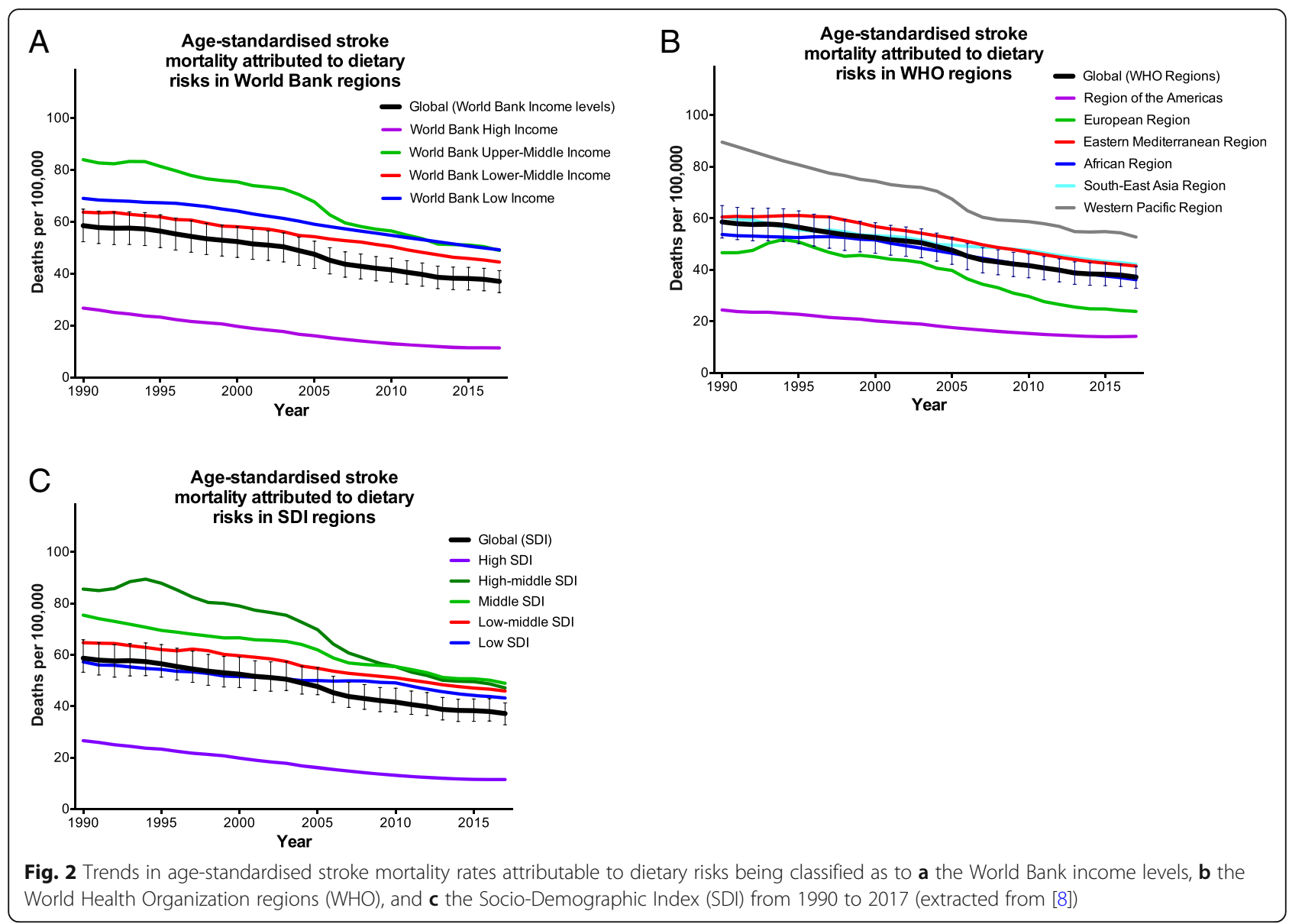

\section{Smoking}

Tobacco smoking became the fifth leading predictor of stroke in 2017 (Table 4). From 1990 to 2017, there was a $41.6 \%$ decrease in the age-standardised global stroke mortality attributable to tobacco, which varied from a $30.5 \%$ decrease in LICs to a $72.3 \%$ decrease in HICs (Table 3). However, the highest tobacco-attributed stroke mortality rate has been observed in UMICs from 1990 to 2017, which holds the third place, after hypertension and dietary risks, among all modifiable risk factors in this specific income category. Overall, there is a 4.5 -fold difference in rates of stroke mortality attributable to tobacco between the mostaffected and the least-affected income category, from 18.9 per 100,000 in UMICs to 4.2 per 100,000 in HICs.

\section{Air pollution}

Air pollution is globally the sixth leading cause of stroke death with no change in its rank from 1990 to 2017 (Table 4). Its attributable risk is higher in regions with lower SES. There is a downward trend in stroke mortality attributable to air pollution in all regions of the World Bank income levels, which is steep in LICs and in UMICs. This suggests improved general awareness in these regions, in particular. However, with regard to the
SDI classification, educational attainment and society population do not seem to affect the attributable risk of stroke mortality due to air pollution (Table 4). Overall, the age-standardised global stroke mortality rate attributable to air pollution has decreased by $62.3 \%$ from 1990 to 2017 , which varied from $33.0 \%$ decrease in LICs to $47.4 \%$ decrease in UMICs (Table 3). Overall, there is a 1.9-fold difference in rates of stroke mortality attributable to air pollution between the most-affected and the least-affected income category, from 16.2 per 100,000 in LICs to 8.6 per 100,000 in HICs.

In 2017, ambient particulate matter pollution and household air pollution from solid fuels were globally responsible for 10.5 and 5.9 million stroke-related DALYs lost and 444.9 and 231.8 thousand stroke-related deaths, respectively (Table 6). Based on the age-standardised rates per 100,000 people worldwide, a third of the 2017 air pollution-related stroke mortality was attributable to household air pollution and two thirds of it was attributable to ambient air pollution. The portion of household air pollution dominates in LICs; it dramatically decreases in wealthier societies, in particular, UMICs and HICs, and increases in LICs and LMICs. Household air pollution is more common in females. Furthermore, in 2017, the attributable risk of 
Table 5 Fractions of stroke incidence, prevalence, mortality, and DALYs lost in different age-groups and income levels in 2017

\begin{tabular}{|c|c|c|c|c|c|}
\hline & LICs & LMICS & HMICS & $\mathrm{HICs}$ & Globa \\
\hline \multicolumn{6}{|l|}{ Incidence (\%) } \\
\hline$<40$ years & 1.4 & 1.4 & 1 & 1.7 & 1.3 \\
\hline 40-64 years & 10.7 & 11 & 10.9 & 9.8 & 10.9 \\
\hline$\geq 65$ years & 87.8 & 87.5 & 88.1 & 88.5 & 87.8 \\
\hline \multicolumn{6}{|l|}{ Prevalence (\%) } \\
\hline$<$ 40years & 3.1 & 2.8 & 2 & 2.4 & 2.1 \\
\hline 40-64 years & 18.7 & 18.5 & 16 & 13.3 & 15.2 \\
\hline$\geq 65$ years & 78.2 & 78.7 & 82 & 84.3 & 82.7 \\
\hline \multicolumn{6}{|l|}{ Mortality (\%) } \\
\hline$<40$ years & 0.8 & 0.5 & 0.3 & 0.2 & 0.5 \\
\hline 40-64 years & 4.4 & 3.9 & 2.7 & 1.5 & 3.4 \\
\hline$\geq 65$ years & 94.8 & 95.6 & 97 & 98.3 & 96 \\
\hline \multicolumn{6}{|l|}{ DALYs (\%) } \\
\hline$<40$ years & 6.3 & 4 & 2.5 & 2.6 & 4.4 \\
\hline 40-64 years & 15.4 & 14.8 & 11.1 & 8.4 & 13.2 \\
\hline$\geq 65$ years & 78.2 & 81.2 & 86.4 & 88.9 & 82.4 \\
\hline
\end{tabular}

$D A L Y s$ disability-adjusted life years, HICs high-income countries, LICs lowincome countries, LMICs lower-middle-income countries, UMICs upper-middle-income countries

Data were extracted from [8]

haemorrhagic stroke was almost double that of ischaemic stroke worldwide.

\section{Alcohol use}

The global rank of stroke mortality related to alcohol drinking rose from the eighth rank in 1990 to the seventh in 2017 (Table 4). Although the age-standardised global stroke mortality rate attributable to alcohol use has declined by 13.5\% from 1990 to 2017, particularly in HICs (70.7\%), its risk increased in both LMICs (33.5\%) and UMICs (3.8\%) (Table 3). In 1995, the rate of alcohol use had moved up one step to the seventh rank with no change in rankings later on. With regard to sex, no change in rankings of these predictors was observed in men during the 25-year period, while in women, the age-standardised rate of alcohol use has shown a two-step decline to the seventh rank in 2017. Overall, there is a 5.3-fold difference in rates of stroke mortality attributable to alcohol use between the most-affected and the least-affected income category, from 11.1 per 100 , 000 in UMICs to 2.1 per 100,000 in HICs.

\section{Hypercholesterolemia}

Hypercholesterolemia, particularly high LDL-C, became globally the eighth most important indicator of stroke mortality in 2017 (Table 4). From 1990 to 2017, the agestandardised global stroke mortality rate attributable to hypercholesterolemia decreased by $38.4 \%$, which varied from $16.4 \%$ decrease in LICs to $64.0 \%$ decrease in HICs
(Table 3). In societies of higher SES, it stands at a higher rank with regard to stroke mortality. Overall, there is a 2.8 -fold difference in rates of stroke mortality attributable to hypercholesterolemia between the most-affected and the least-affected income category, from 9.2 per 100 , 000 in UMICs to 3.3 per 100,000 in HICs.

\section{Low physical activity}

Low physical activity has globally remained the ninth modifiable indicator of stroke mortality since 1990, which is almost constant through the varying SES regions (Table 4). SES can be a factor in determining levels and types of physical activity, as well as facilities accessed. Nevertheless, despite the proven association of low physical activity with stroke, there is a $37.3 \%$ decline in its attributable risk of the age-standardised global stroke mortality, varying between $22.4 \%$ in LICs and $59.8 \%$ in HICs (Table 3). Overall, there is a 2.6 -fold difference in rates of stroke mortality attributable to low physical activity between the most-affected and the least-affected income category, from 5.1 per 100,000 in UMICs to 2.0 per 100,000 in HICs.

\section{Non-modifiable predictors of stroke mortality \\ Age}

Ageing is regarded as the most important predictor of stroke incidence and mortality, and thus, their rates increase by age (Fig. 3). Older individuals with lower SES have higher stroke incidence [14] and mortality rates [15]. Earlier, it was estimated that approximately $90 \%$ of strokes occur above age 65 years, $75 \%$ of which is $>75$ years [16]. By 2017, these rates have slightly changed: $88 \%$ of global strokes occur above age 65 years, $72 \%$ of which is $>75$ years (Table 5 ). This suggests that the age of stroke occurrence is declining and its incidence rate is increasing at younger ages. Compared to HICs, stroke prevalence at younger ages is higher in LICs (15.7\% vs. $21.8 \%$ before age 65 years; $17.3 \%$ globally). In 2017, $96 \%$ of stroke-related deaths happened above age 65 years, $86 \%$ of which was $>75$ years. There is also an almost twofold difference in DALYs lost at younger ages (i.e. lower than 65 years) between LICs and HICs (21.7\% vs. $11.1 \%)$.

\section{Sex}

Risk of stroke and the risk factors differ between men and women, with almost no change in their attributed risk of stroke mortality from 1990 to 2017, particularly for tobacco use, alcohol use, obesity, and air pollution (Table 2, Additional file 3: Table S2, and Table 7). Underlying aetiology, risk factors, incidence, and outcomes of stroke vary substantially between men and women $[17,18]$. Stroke occurs earlier (at 68.5 vs. 73.0 


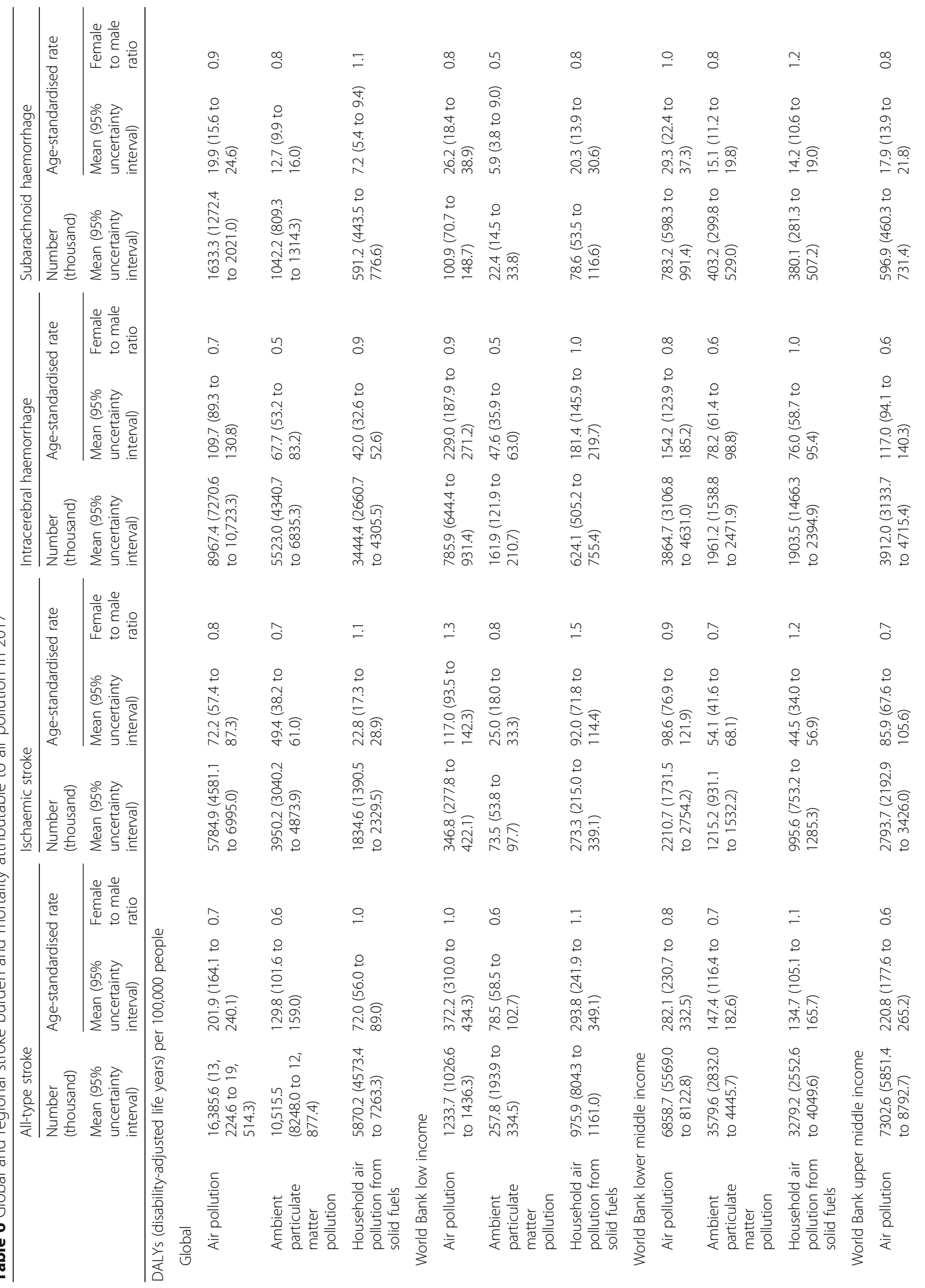




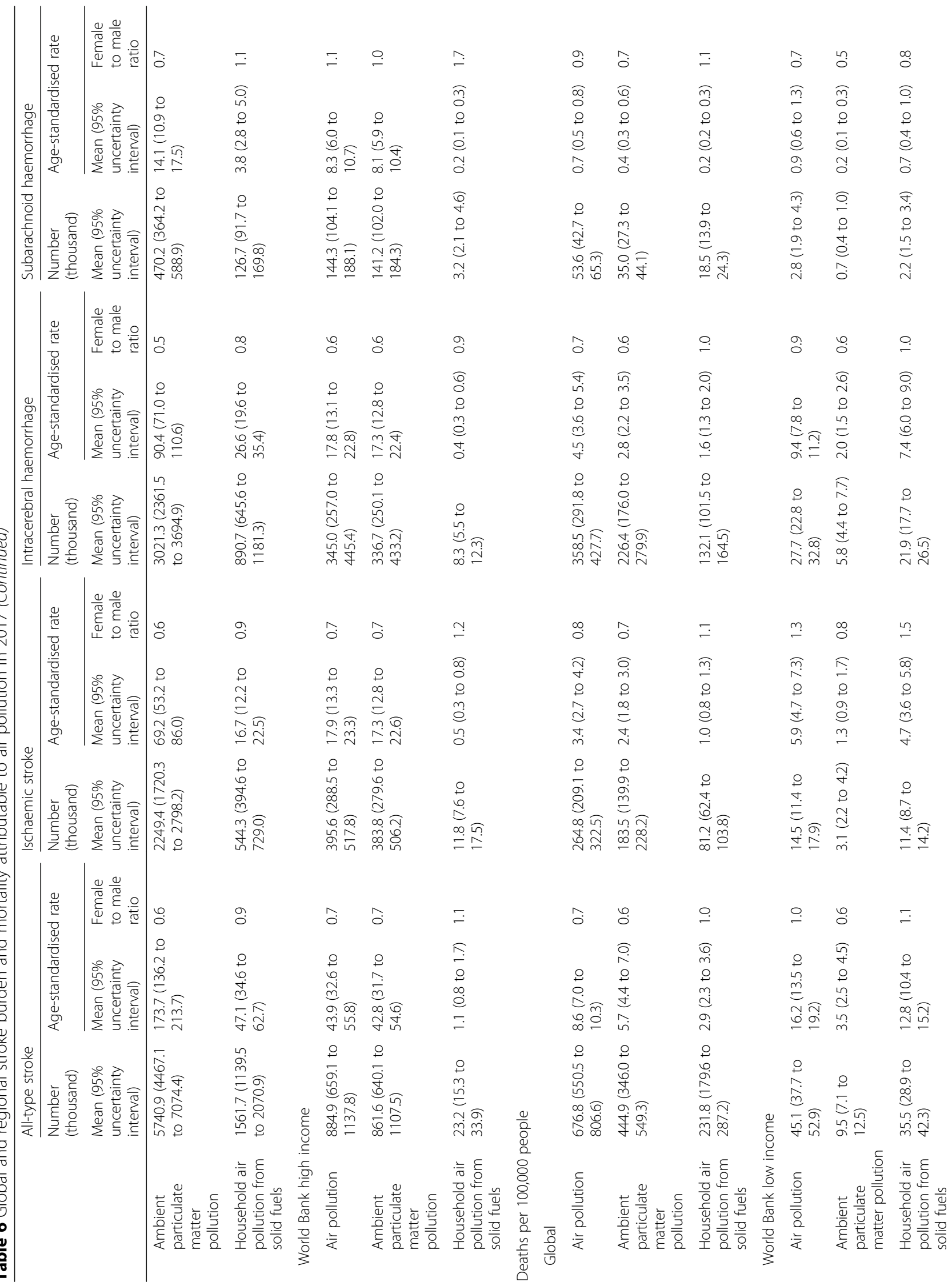




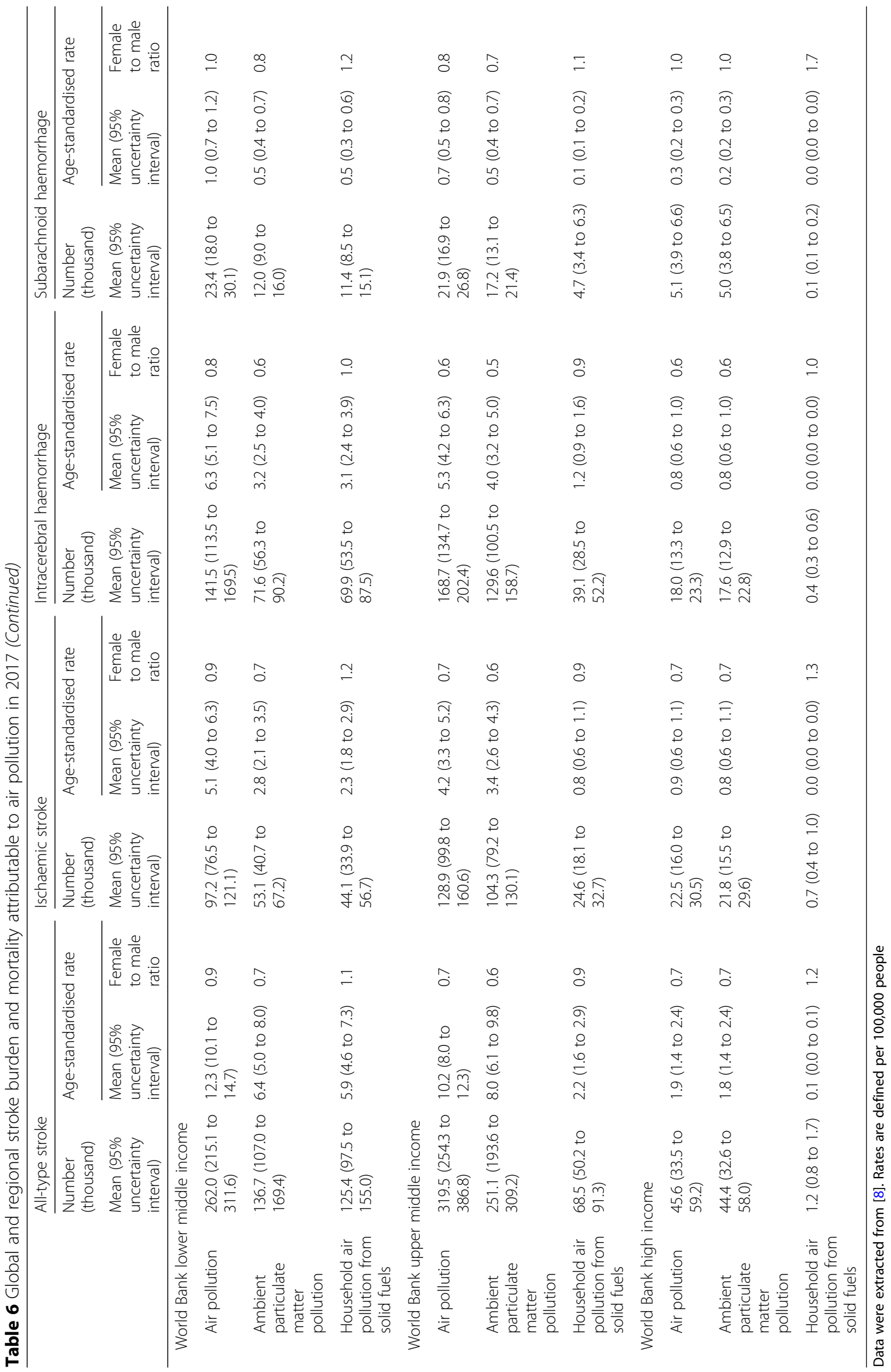




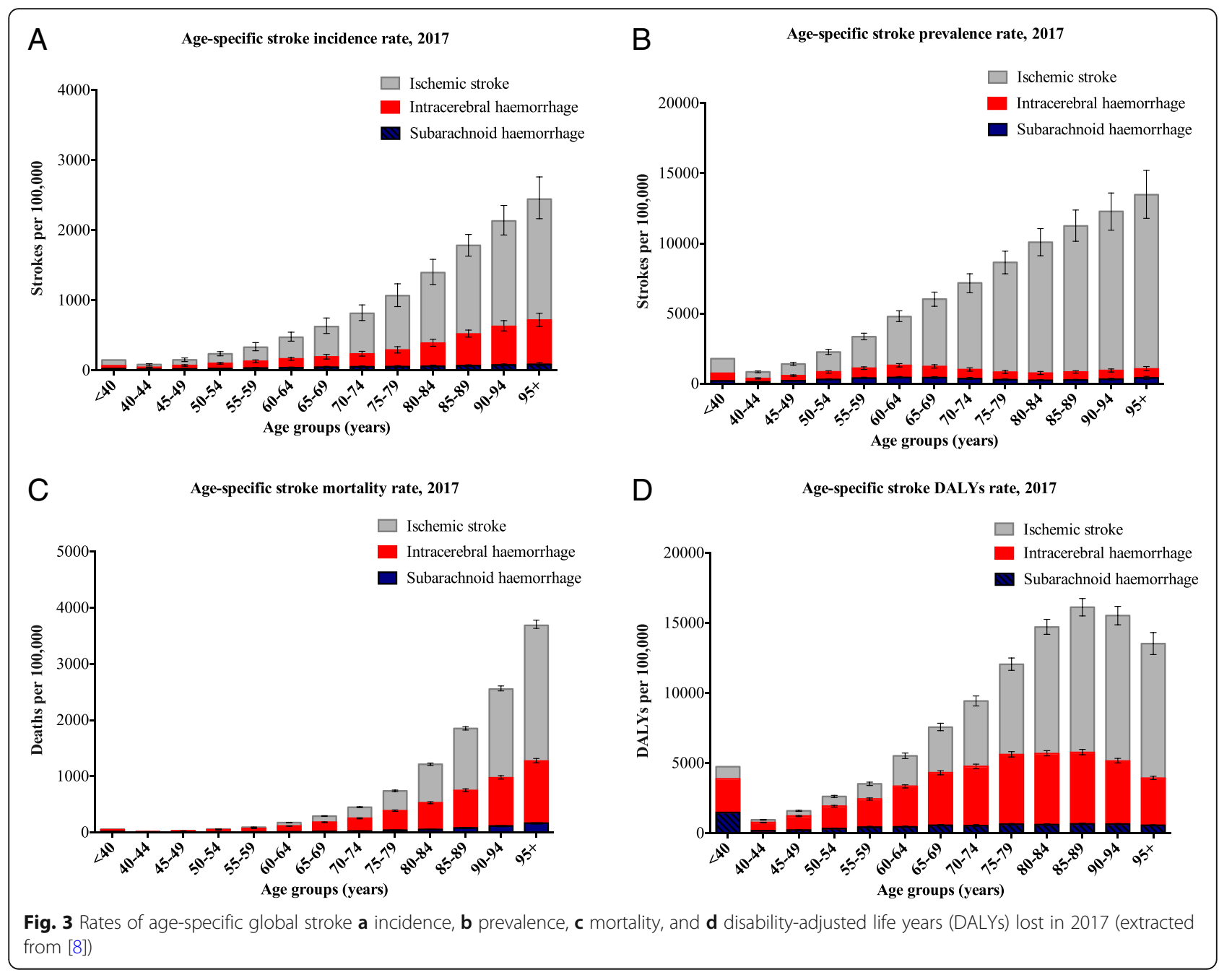

years) and more frequently in men (incidence 33\% higher, and prevalence $41 \%$ higher) than in women [19], while women have more severe strokes resulting in a higher 1-month stroke fatality ( $25 \%$ vs. $20 \%)$ [20]. Our results suggest a pivotal role for SES in sex-stratified age-standardised stroke mortality rates (Table 8).

\section{Discussion}

The results of the current study add to the body of evidence on existing disparities, gaps, and hurdles in stroke research, practice, and educational endeavours in different socioeconomic classes, countries, and regions. It appears that the age-standardised rate of stroke incidence and mortality is decreasing in all regions of varying SES (based on income-level or SDI), although more rapidly in wealthier societies. This influence is largely driven through commonly known modifiable stroke risk factors. In 2017, high systolic blood pressure and dietary risks were the top leading causes of stroke-related deaths and worldwide burden.
Among the 11 modifiable risk factors, alcohol had five times higher association with haemorrhagic strokes than ischaemic strokes. Further, the rates of stroke mortality and burden were significantly higher in lower SES regions compared with HICs. Likewise, the age-standardised stroke mortality attributable to potentially modifiable risk factors is declining in almost all regions, except for obesity and alcohol use. LICs hold the worst attributable risk of stroke mortality for hypertension, dietary habits, diabetes, and air pollution, and UMICs hold the worst attributable risk of stroke mortality for obesity, tobacco use, alcohol use, hypercholesterolemia, and low physical activity. Still, HICs have almost threefold lower rates of stroke mortality attributable to modifiable risk factors compared to all other income categories.

According to the last report of the global, regional, and national burden of neurological disorders, there has been a significant reduction in the age-standardised prevalence of stroke (10\% decrease) and death $(30 \%$ 
Table 7 Differences in the ranks of age-standardised stroke mortality rate attributable to dietary risk factors being stratified by sex in 1990 and 2017 (extracted from [8])

\begin{tabular}{|c|c|c|c|c|c|}
\hline \multirow{3}{*}{\multicolumn{2}{|c|}{$\begin{array}{c}\text { Rank } \\
1\end{array}$}} & \multicolumn{4}{|c|}{$\begin{array}{c}\text { Global age-standardised stroke } \\
\text { mortality }\end{array}$} \\
\hline & & \multicolumn{2}{|c|}{ Male } & \multicolumn{2}{|c|}{ Female } \\
\hline & & 1990 rank & 2017 rank & 1990 rank & 2017 rank \\
\hline Hypertension & & 1 & 1 & 1 & 1 \\
\hline Dietary risks & & 2 & 2 & 2 & 2 \\
\hline Tobacco & & 3 & 3 & 6 & 7 \\
\hline $\begin{array}{l}\text { Impaired glucose } \\
\text { tolerance }\end{array}$ & & 4 & 4 & 3 & 3 \\
\hline Alcohol use & & 5 & 5 & 9 & 9 \\
\hline Air pollution & & 6 & 7 & 5 & 5 \\
\hline Obesity & & 7 & 6 & 4 & 4 \\
\hline Hypercholesterolemia & & 8 & 8 & 7 & 6 \\
\hline Low physical activity & & 9 & 9 & 8 & 8 \\
\hline
\end{tabular}

decrease) from 1990 to 2015 [1]. By 2017, according to the present study, the age-standardised rates of global stroke prevalence and mortality exhibited a 3\% increase and a $33 \%$ decrease, respectively. This reflects the evolving nature of the GBD data and the changes in stroke burden worldwide.

Based on the results of a meta-analysis of 12 population-based cohorts and case-control studies mainly in HICs, despite some limitations, reduced SES could explain more than 30\% (95\% confidence interval 16-48) of the stroke risk irrespective of classical vascular risk factors [21]. Based on another meta-analysis by Kerr et al. [21], blood pressure, smoking, diabetes, lipids, atrial fibrillation, history of vascular disease, obesity, and physical activity were overall associated with an additional $30-40 \%$ risk.

Table 8 Difference in ranks of age-standardised stroke mortality rate attributable to modifiable risk factors being stratified by sex in the World Bank (WB) low-income countries (LICS), lower-middle-income countries (LMICs), upper-middle-income countries (UMICS), and high-income countries (HICS) in 1990 and 2017 (extracted from [8])

\begin{tabular}{|c|c|c|c|c|c|c|c|c|c|c|c|c|c|}
\hline & \multirow{3}{*}{$\begin{array}{c}\text { Rank } \\
- \\
-10\end{array}$} & \multicolumn{12}{|c|}{$\begin{array}{l}\text { Age-standardised stroke mortality related to modifiable risk factors, } \\
\text { according to gender and World Bank (WB) income levels (2017), } \\
\text { (death rate per 100,000) }\end{array}$} \\
\hline & & \multicolumn{3}{|c|}{ WB LICs } & \multicolumn{3}{|c|}{ WB LMICs } & \multicolumn{3}{|c|}{ WB UMICs } & \multicolumn{3}{|c|}{ WB HICs } \\
\hline & & $\begin{array}{l}\text { Both } \\
\text { sexes }\end{array}$ & Male & Female & $\begin{array}{l}\text { Both } \\
\text { sexes }\end{array}$ & Male & Female & $\begin{array}{l}\text { Both } \\
\text { sexes }\end{array}$ & Male & Female & $\begin{array}{c}\text { Both } \\
\text { sexes }\end{array}$ & Male & Female \\
\hline Hypertension & & 1 & 1 & 1 & 1 & 1 & 1 & 1 & 1 & 1 & 1 & 1 & 1 \\
\hline Dietary risks & & 2 & 2 & 2 & 2 & 2 & 2 & 2 & 2 & 2 & 2 & 2 & 2 \\
\hline $\begin{array}{l}\text { Impaired glucose } \\
\text { tolerance }\end{array}$ & & 3 & 4 & 3 & 3 & 3 & 3 & 4 & 5 & 4 & 3 & 3 & 3 \\
\hline Air pollution & & 4 & 7 & 4 & 6 & 6 & 5 & 7 & 7 & 6 & 9 & 8 & 8 \\
\hline Obesity & & 5 & 6 & 5 & 4 & 5 & 4 & 5 & 6 & 3 & 4 & 4 & 4 \\
\hline Tobacco & & 6 & 3 & 7 & 5 & 4 & 7 & 3 & 3 & 7 & 5 & 5 & 6 \\
\hline Hypercholesterolemia & & 7 & 8 & 6 & 7 & 8 & 6 & 8 & 8 & 5 & 6 & 7 & 5 \\
\hline Low physical activity & & 8 & 9 & 8 & 9 & 9 & 8 & 9 & 9 & 8 & 8 & 9 & 7 \\
\hline Alcohol use & & 9 & 5 & 9 & 8 & 7 & 9 & 6 & 4 & 9 & 7 & 6 & 9 \\
\hline
\end{tabular}


Nevertheless, more population-based studies have been conducted since then, which improved our understanding of the predicting factors [22]. For instance, an Australian population-based study of 3077 subjects with incident stroke between 1995 and 2003 proposed that improving the SES of the most deprived inhabitants could prevent up to one fifth of strokes [23].

The disparity between stroke outcomes in LMICs and HICs can be partly explained by a combination of varying levels of general health awareness, access to healthcare, and preventative strategies starting from childhood $[24,25]$. Stroke management is also challenging. Excessively increasing trends of urbanisation, pollution, smoking, obesity, less physical activity, having unhealthy diets, and ageing, particularly in LMICs, along with a much larger population with restricted access to health care may partially predict the increasing rates of stroke in these countries. This alarming increase necessitates a thorough investigation of all possible reasons, including SES determinants. Accordingly, despite many recent studies in LICs and LMICs [26-36] (Additional file 5: Table S4), we cannot confidently generalise the association of common vascular risk factors with the risk and outcome of stroke in LICs and LMICs. There used to be a ten-fold difference in rates of stroke mortality and burden between the most-affected and the least-affected countries [37]. Currently, based on our findings, this has decreased to almost threefold, which is suggestive of a substantial improvement in healthcare, preventive measures, and therapeutic outcomes. However, we should acknowledge the scantiness of population-based studies in LICs and LMICs compared to UMICs and HICs.

According to the GBD 2015 [38], there are regular shifts in stroke-related cause of death composition and population age structure with rising SDI (Table 4). Moreover, ischaemic heart disease, stroke, and diabetes are among the leading causes of premature mortality, measured as years of life lost, in most SES regions based on SDI [38]. The present study highlighted the necessity of country-specific and SES-stratified quantification of the risk of stroke attributable to each factor [22].

\section{Conclusions}

Almost half of stroke-related mortality may be attributable to modifiable risk factors (i.e. hypertension, diabetes, dietary risks, impaired glucose intolerance, obesity, smoking, air pollution, alcohol use, hypercholesterolemia, and physical inactivity), which are mostly the outcome of poor clinical management, limited access to health care, and late detection of underlying risk factors. This necessitates allocation of resources to those modifiable risk factors with the highest impact on stroke in each SES-region. Moreover, social and economic policies to reduce inequalities in stroke care should become a health priority, particularly in less wealthy countries. These policies should focus on treating early predisposing factors and on educational programmes from childhood, which have long-lasting impacts on adulthood health. Likewise, improving worldwide primary healthcare services may have an important impact on post-stroke outcomes. It is essential to improve stroke awareness among socioeconomically deprived individuals and societies and provide equitable post-stroke medical care.

\section{Additional files}

Additional file 1: Text S1. Definition of Socioeconomic status; Data gathering and search strategy; Study selection, data extraction, and analysis; Supplements to sections on various risk factors. (DOCX 40 kb)

Additional file 2: Table S1. Absolute numbers and rates of stroke mortality and burden attributable to modifiable risk factors. (XLSX $534 \mathrm{~kb}$ )

Additional file 3: Table S2. Sex differences in stroke-related deaths and burden attributable to modifiable risk factors. (XLSX $501 \mathrm{~kb}$ )

Additional file 4: Table S3. Age-standardised rates of stroke mortality and burden attributable to behavioural, environmental, and metabolic risks. (XLSX 501 kb)

Additional file 5: Table S4. Socioeconomic Status and Stroke Outcome in Low- and Middle-Income Countries. (DOCX 132 kb)

\section{Abbreviations}

GBD: The Global Burden of Diseases, Injuries, and Risk Factors Study; HI: High-income; HICs: High-income countries; LICs: Low-income countries; LMICs: Lower-middle-income countries; SDI: Socio-demographic Index; SES: Socioeconomic status; UI: Uncertainty interval; UMICs: Upper-middleincome countries; WB: The World Bank

\section{Acknowledgements}

Not applicable.

\section{Authors' contributions}

All authors contributed to the study concept and design. AAv was responsible for the search design, literature search, data analysis, interpretation of data, and preparation of the first draft of the manuscript, figures, and tables. AAv and HD were responsible for the literature review. $A A v$ and GS were responsible for the data extraction and acquisition of data. AAv, HD, MDN, and JDS were responsible for the draft of Additional file 1: Text S1. JDS was responsible for the proofreading. All authors critically revised the manuscript for relevant intellectual content. All authors read and approved the final manuscript.

Funding

None.

Availability of data and materials

The datasets generated and/or analysed during the current study are available in [8].

Ethics approval and consent to participate

All data were publicly available, no patient contact was made, and no individual identifiers were required. Therefore, ethical approval for the study was not required.

Consent for publication

Not applicable.

Competing interests

The authors declare that they have no competing interests. 


\section{Author details}

Department of Neurology, Ghaem Hospital, School of Medicine, Mashhad University of Medical Sciences, Mashhad, Iran. ${ }^{2}$ Neurobiology Research Center, Shahid Beheshti University of Medical Sciences, Tehran, Iran. ${ }^{3}$ Department of Neurology and Stroke Unit, San Camillo de' Lellis General District Hospital, Rieti, Italy. ${ }^{4}$ Department of Epidemiology and Biostatistics, Schulich School of Medicine \& Dentistry, Western University, London, Ontario, Canada. ${ }^{5}$ Department of Family Medicine, Schulich School of Medicine \& Dentistry, Western University, London, Ontario, Canada. ${ }^{6}$ Department of Population Health, Luxembourg Institute of Health, Strassen, Luxembourg. ${ }^{7}$ Stroke Program, Department of Neurology, School of Medicine, University of Texas Health Science Center, San Antonio, TX, USA. ${ }^{8}$ Health Policy Research Center, Institute of Health, Shiraz University of Medical Sciences, Shiraz, Iran. ${ }^{9}$ Clinical Neurology Research Center, Shiraz University of Medical Sciences, Shiraz, Iran. ${ }^{10}$ Department of Psychiatry and Behavioural Neurosciences, Western University, London, Ontario, Canada. ${ }^{11}$ Department of Psychiatry, Mashhad University of Medical Sciences, Mashhad, Iran. ${ }^{12}$ Stroke Prevention \& Atherosclerosis Research Centre, Robarts Research Institute, Western University, Siebens-Drake Building, 1400 Western Rd, London, Ontario N6G 2V4, Canada. ${ }^{13}$ Department of Clinical Neurological Science, Western University, London, Ontario, Canada. ${ }^{14}$ Division of Clinical Pharmacology, Western University, London, Ontario, Canada.

\section{Received: 18 February 2019 Accepted: 25 July 2019} Published online: 24 October 2019

\section{References}

1. GBD 2015 Neurological Disorders Collaborator Group. Global, regional, and national burden of neurological disorders during 1990-2015: a systematic analysis for the Global Burden of Disease Study 2015. Lancet Neurol. 2017. 16:877-97. https://doi.org/10.1016/S1474-4422(17)30299-5.

2. World Health Organization. Projections of mortality and causes of death, 2015 and 2030. Health statistics and information systems. 2013. http://www. who.int/entity/healthinfo/global_burden_disease/GHE_DthGlobal_Proj_ 2015 2030.xIs?ua=1. Accessed 21 June 2016.

3. World Bank Country and Lending Groups. The World Bank. 2017. https:// datahelpdesk.worldbank.org/knowledgebase/articles/906519. Accessed 1 Dec 2017

4. Aslanyan S, Weir CJ, Lees KR, Reid JL, Mclnnes GT. Effect of area-based deprivation on the severity, subtype, and outcome of ischemic stroke. Stroke. 2003;34:2623-8. https://doi.org/10.1161/01.STR.0000097610.12803.D7.

5. Wu SH, Woo J, Zhang X-H. Worldwide socioeconomic status and stroke mortality: an ecological study. Int J Equity Health. 2013;12:42. https://doi. org/10.1186/1475-9276-12-42

6. Feigin $\mathrm{VL}$, Forouzanfar MH, Krishnamurthi R, Mensah GA, Connor M, Bennett DA, et al. Global and regional burden of stroke during 1990-2010: findings from the Global Burden of Disease Study 2010. Lancet. 2014;383:245-54. https://doi.org/10.1016/S0140-6736(13)61953-4

7. Buttar HS, Li T, Ravi N. Prevention of cardiovascular diseases: Role of exercise, dietary interventions, obesity and smoking cessation. Exp Clin Cardiol. 2005;10:229-49.

8. Global Burden of Disease Collaborative Network. Global Burden of Disease Study 2017 (GBD 2017) Results. Seattle, United States: Institute for Health Metrics and Evaluation (IHME). 2018. http://ghdx.healthdata.org/gbd-resultstool. Accessed 19 May 2019.

9. GBD 2017 Mortality Collaborators. Global, regional, and national age-sexspecific mortality and life expectancy, 1950-2017: a systematic analysis for the Global Burden of Disease Study 2017. Lancet. 2018;392:1684-735. https://doi.org/10.1016/S0140-6736(18)31891-9.

10. GBD 2017 Disease and Injury Incidence and Prevalence Collaborators. Global, regional, and national incidence, prevalence, and years lived with disability for 354 diseases and injuries for 195 countries and territories, 19902017: a systematic analysis for the Global Burden of Disease Study 2017. Lancet. 2018:392:1789-858. https://doi.org/10.1016/S0140-6736(18)32279-7.

11. GBD 2016 Neurology Collaborators. Global, regional, and national burden of neurological disorders, 1990-2016: a systematic analysis for the Global Burden of Disease Study 2016. Lancet Neurol. 2019;18:459-80. https://doi. org/10.1016/S1474-4422(18)30499-X

12. GBD 2013 Mortality and Causes of Death Collaborators. Global, regional, and national age-sex specific all-cause and cause-specific mortality for 240 causes of death, 1990-2013: a systematic analysis for the Global Burden of
Disease Study 2013. Lancet. 2015;385:117-71. https://doi.org/10.1016/S014 0-6736(14)61682-2.

13. Clarke P, O'Malley PM, Johnston LD, Schulenberg JE. Social disparities in BMI trajectories across adulthood by gender, race/ethnicity and lifetime socioeconomic position: 1986-2004. Int J Epidemiol. 2009;38:499-509. https://doi. org/10.1093/ije/dyn214

14. Avendano M, Kawachi I, Van Lenthe F, Boshuizen HC, Mackenbach JP, Van den Bos GAM, et al. Socioeconomic status and stroke incidence in the US elderly: the role of risk factors in the EPESE study. Stroke. 2006;37:1368-73. https://doi.org/10.1161/01.STR.0000221702.75002.66.

15. Bassuk SS, Berkman LF, Amick BC. Socioeconomic status and mortality among the elderly: findings from four US communities. Am J Epidemiol. 2002;155:520-33.

16. Centers for Disease Control and Prevention (CDC). State-specific mortality from stroke and distribution of place of death--United States, 1999. MMWR Morb Mortal Wkly Rep. 2002;51:429-33.

17. Poorthuis MHF, Algra AM, Algra A, Kappelle LJ, Klijn CJM. Female- and malespecific risk factors for stroke: a systematic review and meta-analysis. JAMA Neurol. 2017;74:75-81. https://doi.org/10.1001/jamaneurol.2016.3482.

18. Persky RW, Turtzo LC, McCullough LD. Stroke in women: disparities and outcomes. Curr Cardiol Rep. 2010;12:6-13. https://doi.org/10.1007/s11886009-0080-2.

19. Appelros $P$, Stegmayr B, Terént A. Sex differences in stroke epidemiology: a systematic review. Stroke. 2009:40:1082-90. https://doi.org/10.1161/ STROKEAHA.108.540781.

20. Reeves MJ, Bushnell CD, Howard G, Gargano JW, Duncan PW, Lynch G, et al. Sex differences in stroke: epidemiology, clinical presentation, medical care, and outcomes. Lancet Neurol. 2008;7:915-26. https://doi.org/10.1016/S1474-4422(08)70193-5.

21. Kerr GD, Slavin H, Clark D, Coupar F, Langhorne P, Stott DJ. Do vascular risk factors explain the association between socioeconomic status and stroke incidence: a meta-analysis. Cerebrovasc Dis. 2011;31:57-63. https://doi.org/10.1159/000320855.

22. Ferrario MM, Veronesi G, Kee F, Chambless LE, Kuulasmaa K, Jørgensen T, et al. Determinants of social inequalities in stroke incidence across Europe: a collaborative analysis of 126635 individuals from 48 cohort studies. J Epidemiol Community Health. 2017;71:1210-6. https://doi.org/10.1136/ jech-2017-209728.

23. Heeley EL, Wei JW, Carter K, Islam MS, Thrift AG, Hankey GJ, et al. Socioeconomic disparities in stroke rates and outcome: pooled analysis of stroke incidence studies in Australia and New Zealand. Med J Aust. 2011:195:10-4.

24. Liu L, Xue F, Ma J, Ma M, Long Y, Newschaffer CJ. Social position and chronic conditions across the life span and risk of stroke: a life course epidemiological analysis of 22,847 American adults in ages over 50. Int J Stroke. 2013;8(Suppl A100):50-5. https://doi.org/10.1111/j.1747-4949.2012.00927.x.

25. Becher H, Palm F, Aigner A, Safer A, Urbanek C, Buggle F, et al. Socioeconomic conditions in childhood, adolescence, and adulthood and the risk of ischemic stroke. Stroke. 2016;47:173-9. https://doi.org/10.1161/ STROKEAHA.115.011523.

26. Copstein L, Fernandes JG, Bastos GAN. Prevalence and risk factors for stroke in a population of Southern Brazil. Arq Neuropsiquiatr. 2013;71:294-300.

27. Engels T, Baglione Q, Audibert M, Viallefont A, Mourji F, El Alaoui Faris $M$, et al. Socioeconomic status and stroke prevalence in Morocco: results from the Rabat-Casablanca study. PLoS ONE. 2014;9:e89271. https://doi.org/10.1371/journal.pone.0089271.

28. Fitzpatrick AL, Ngo QV, Ly KA, Ton TGN, Longstreth WT, Vo TT, et al. Symptoms and risk factors for stroke in a community-based observational sample in Viet Nam. J Epidemiol Glob Health. 2012;2:155-63. https://doi. org/10.1016/j.jegh.2012.06.001.

29. Jin H, Zhu S, Wei JW, Wang J, Liu M, Wu Y, et al. Factors associated with prehospital delays in the presentation of acute stroke in urban China. Stroke. 2012;43:362-70. https://doi.org/10.1161/ STROKEAHA.111.623512

30. Kamal AK, Rasheed A, Mehmood K, Murtaza M, Zaidi M, Khan M, et al. Frequency and determinants of intracranial atherosclerotic stroke in urban Pakistan. J Stroke Cerebrovasc Dis. 2014;23:2174-82. https://doi.org/10.1016/ j.jstrokecerebrovasdis.2014.04.003.

31. Kumar A, Prasad M, Kathuria P, Nair P, Pandit AK, Sahu JK, et al. Low socioeconomic status is an independent risk factor for ischemic stroke: a case-control study in North Indian population. Neuroepidemiology. 2015:44: 138-43. https://doi.org/10.1159/000374118. 
32. Menon J, Vijayakumar N, Joseph JK, David PC, Menon MN, Mukundan S, et al. Below the poverty line and non-communicable diseases in Kerala: The Epidemiology of Non-communicable Diseases in Rural Areas (ENDIRA) study. Int J Cardiol. 2015;187:519-24. https://doi.org/10.1016/.j.jcard.2015.04.009.

33. Pandian JD, Jyotsna R, Singh R, Sylaja PN, Vijaya P, Padma MV, et al. Premorbid nutrition and short term outcome of stroke: a multicentre study from India. J Neurol Neurosurg Psychiatr. 2011;82:1087-92. https://doi.org/10.1136/jnnp.2010.233429.

34. Tang X, Laskowitz DT, He L, Østbye T, Bettger JP, Cao Y, et al. Neighborhood socioeconomic status and the prevalence of stroke and coronary heart disease in rural China: a population-based study. Int I Stroke. 2015;10:388-95. https://doi.org/10.1111/ijs.12343.

35. Xu F, Ah Tse L, Yin X, Yu IT, Griffiths S. Impact of socio-economic factors on stroke prevalence among urban and rural residents in Mainland China. BMC Public Health. 2008;8:170. https://doi.org/10.1186/1471-2458-8-170.

36. Zhou G, Liu X, Xu G, Liu X, Zhang R, Zhu W. The effect of socioeconomic status on three-year mortality after first-ever ischemic stroke in Nanjing, China. BMC Public Health. 2006;6:227. https://doi. org/10.1186/1471-2458-6-227.

37. Johnston SC, Mendis S, Mathers CD. Global variation in stroke burden and mortality: estimates from monitoring, surveillance, and modelling. Lancet Neurol. 2009;8:345-54. https://doi.org/10.1016/S1474-4422(09)70023-7.

38. GBD. Global, regional, and national life expectancy, all-cause mortality, and cause-specific mortality for 249 causes of death, 1980-2015: a systematic analysis for the Global Burden of Disease Study 2015. Lancet. 2016;388: 1459-544. https://doi.org/10.1016/50140-6736(16)31012-1.

\section{Publisher's Note}

Springer Nature remains neutral with regard to jurisdictional claims in published maps and institutional affiliations.

Ready to submit your research? Choose BMC and benefit from:

- fast, convenient online submission

- thorough peer review by experienced researchers in your field

- rapid publication on acceptance

- support for research data, including large and complex data types

- gold Open Access which fosters wider collaboration and increased citations

- maximum visibility for your research: over $100 \mathrm{M}$ website views per year

At BMC, research is always in progress.

Learn more biomedcentral.com/submissions 